\title{
Nonlinear and Nonideal Sampling: Theory and Methods
}

\author{
Tsvi G. Dvorkind, Yonina C. Eldar, Senior Member, IEEE, and Ewa Matusiak
}

\begin{abstract}
We study a sampling setup where a continuous-time signal is mapped by a memoryless, invertible and nonlinear transformation, and then sampled in a nonideal manner. Such scenarios appear, for example, in acquisition systems where a sensor introduces static nonlinearity, before the signal is sampled by a practical analog-to-digital converter. We develop the theory and a concrete algorithm to perfectly recover a signal within a subspace, from its nonlinear and nonideal samples. Three alternative formulations of the algorithm are described that provide different insights into the structure of the solution: A series of oblique projections, approximated projections onto convex sets, and quasi-Newton iterations. Using classical analysis techniques of descent-based methods, and recent results on frame perturbation theory, we prove convergence of our algorithm to the true input signal. We demonstrate our method by simulations, and explain the applicability of our theory to Wiener-Hammerstein analog-to-digital hybrid systems.
\end{abstract}

Index Terms-Generalized sampling, interpolation, nonlinear sampling, Wiener-Hammerstein.

\section{INTRODUCTION}

D IGITAL signal processing applications are often concerned with the ability to store and process discrete sets of numbers, which are related to continuous-time signals through an acquisition process. One major goal, which is at the heart of digital signal processing, is the ability to reconstruct continuous-time functions, by properly processing their available samples.

In this paper, we consider the problem of reconstructing a function from its nonideal samples, which are obtained after the signal was distorted by a memoryless (i.e., static), nonlinear, and invertible mapping.

The main interest in this setup stems from scenarios where an acquisition device introduces a nonlinear distortion of amplitudes to its input signal, before sampling by a practical

Manuscript received February 26, 2008; revised July 05, 2008. First published August 29, 2008; current version published November 19, 2008. The associate editor coordinating the review of this manuscript and approving it for publication was Dr. Chong-Meng Samson See. This work was supported in part by the Israel Science Foundation under Grant 1081/07 and by the European Commission in the framework of the FP7 Network of Excellence in Wireless COMmunications NEWCOM++ (Contract 216715).

T. G. Dvorkind is with the Rafael Company, Haifa 2250, Israel (e-mail: dvorkind@gmail.com).

Y. C. Eldar is with the Department of Electrical Engineering, Technion-Israel Institute of Technology, Haifa 32000, Israel (e-mail: yonina@ee.technion.ac.il).

E. Matusiak is with the Faculty of Mathematics, University of Vienna, 1090 Wien, Austria (e-mail: ewa.matusiak@univie.ac.at).

Color versions of one or more of the figures in this paper are available online at http://ieeexplore.ieee.org.

Digital Object Identifier 10.1109/TSP.2008.929872

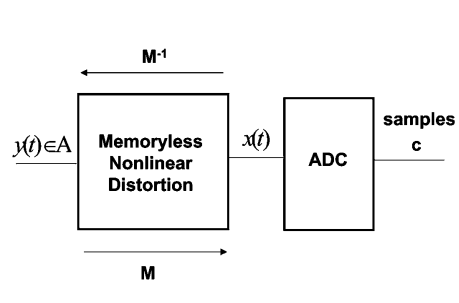

(a)

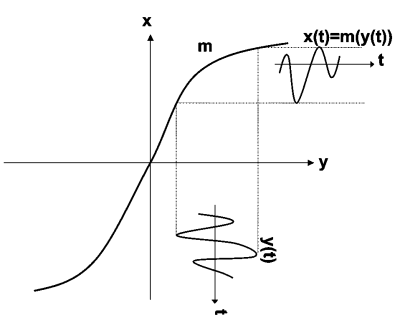

(b)
Fig. 1. (a) Sampling setup. (b) Illustration of the memoryless nonlinear mapping.

analog-to-digital converter (ADC) [see Fig. 1(a)]. Nonlinear distortions appear in a variety of setups and applications of digital signal processing. For example, charge-coupled device (CCD) image sensors introduce nonlinear distortions when excessive light intensity causes saturation [1], [2]. Memoryless nonlinear distortions also appear in the areas of power electronics [3] and radiometric photography [4], [5]. In some cases, nonlinearity is introduced deliberately in order to increase the possible dynamic range of the signal while avoiding amplitude clipping, or damage to the ADC [6]. The goal is then to process the samples in order to recover the original continuous-time function.

The usual assumption in such problems is that the samples are ideal, i.e., they are pointwise evaluations of the nonlinearly distorted, continuous-time signal. Even then, the problem may appear to be hard. For example, nonlinearly distorting a band-limited signal, usually increases its bandwidth. Thus, it might not be obvious how to adjust the sampling rate after the nonlinearity. In [7], for instance, the author seeks sampling rates to reconstruct a band-pass signal, which is transformed by a nonlinear distortion of order at most three. However, as noticed by Zhu [8], oversampling in such circumstances is unnecessary. Assuming the band-limited setting and ideal sampling, Zhu showed that perfect reconstruction of the input signal can be obtained, even if the distorted function is sampled at the Nyquist rate of the input. The key idea is to apply the inverse of the memoryless nonlinearity to the given ideal samples, resulting in ideal samples of the band-limited input signal. Recovery is then straightforward by applying Shannon's interpolation. Unfortunately, in practice, ideal sampling is impossible to implement. A more accurate model considers generalized sampling [9]-[12]. Instead of pointwise evaluations of the continuous-time function, the samples are modeled by a set of inner products between the continuous-time signal and the sampling functions. These sampling functions are related to the linear part of the acquisition process, 
for example they can describe the antialiasing filtering effects of an ADC [9].

In the context of generalized sampling, considerable effort has been devoted to the study of purely linear setups, where nonlinear distortions are absent from the description of the acquisition model. The usual scenario is to reconstruct a function by processing its generalized samples, obtained by a linear and bounded acquisition device. Assuming a shift-invariant setup, the authors of [9] introduce the concept of consistent reconstruction in which a signal is recovered within the reconstruction space, such that when re-injected into the linear acquisition device, the original sample sequence is reproduced. The idea of consistency was then extended to arbitrary Hilbert spaces in [10], [13], and [14]. In some setups, the consistency requirement leads to large error of approximation. Instead, robust approximations were developed in [12], in which, the reconstructed function is optimized to minimize the so called minimax regret criterion, related directly to the squared-norm of the reconstruction error. This approach guarantees a bounded approximation error, however, there as well, the acquisition model is linear.

In this paper, we consider a nonlinear sampling setup, combined with generalized sampling. The continuous-time signal is first nonlinearly distorted and then sampled in a nonideal (generalized) manner. We assume that the nonlinear and nonideal acquisition device is known in advance, and that the samples are noise free. In this general context, we develop the theory to ensure perfect reconstruction, and an iterative algorithm, which is proved to recover the input signal from its nonlinear and generalized samples. The theory we develop leads to simple sufficient conditions on the nonlinear distortion and the spaces involved, that ensure perfect recovery of the input signal. If the signal is not constrained to a subspace, then the problem of perfect recovery becomes ill-posed (see Section III) as there are infinitely many functions which can explain the samples. Therefore, our main effort concerns the practical problem of reconstructing a function within some predefined subspace. For example, the problem may be to reconstruct a band-limited function, though, in this work we are not restricted to the band-limited setup.

Three alternative formulations of the algorithm are developed that provide different insight into the structure of the solution: A series of oblique projections [15], [16], an approximated projections onto convex sets (POCS) method [17], and quasi-Newton iterations [18], [19]. Under some conditions, we show that all three viewpoints are equivalent, and from each formulation we extract interesting insights into the problem.

Our approach relies on linearization, where at each iteration we solve a linear approximation of the original nonlinear sampling problem. To prove convergence of our algorithm we extend some recent results concerning frame perturbation theory [20]. We also apply classical analysis techniques which are used to prove convergence of descent-based methods.

After stating the notations and the mathematical preliminary assumptions in Section II, we formulate our problem in Section III. In Section IV, we prove that under proper conditions, perfect reconstruction of the input signal from its nonlinear and generalized samples is possible. In Section V, we suggest a specific iterative algorithm. The recovery method relies on linearization of the underlying nonlinear problem, and takes on the form of a series of oblique projections. In Section VI, we develop a reconstruction based on the POCS method and show it to be equivalent to the iterative oblique-projections algorithm. In Section VII, we view the linearization approach within the framework of frame perturbation theory. This viewpoint leads to conditions on the nonlinear mapping and the spaces involved, which ensure perfect recovery of the input. In Section VIII, we formulate our algorithm as quasi-Newton iterations, proving convergence of our method. Some practical aspects are discussed in Section IX. Specifically, we explain how the algorithm should be altered, if some of the mathematical preliminary assumptions do not hold in practice. We also show how to apply our results to acquisition devices that are modeled by a Wiener-Hammerstein system [21]. Simulation results are provided in Section X. Finally, in Section XI, we conclude and suggest future directions of research. Some of the mathematical derivations are provided within the appendixes.

\section{NotATIONS AND MATHEMATICAL PRELIMINARIES}

We denote continuous-time signals by bold lowercase letters, omitting the time dependence, when possible. The elements of a sequence $c \in l_{2}$ will be written with square brackets, e.g., $c[n]$. Operators are denoted by upper case letters. The operator $P_{\mathcal{A}}$ represents the orthogonal projection onto a closed subspace $\mathcal{A}$, and $\mathcal{A}^{\perp}$ is the orthogonal complement of $\mathcal{A}$. $P_{\mathcal{A}_{1}, \mathcal{A}_{2}}$ stands for an oblique projection operator [15], [16], with range space $\mathcal{A}_{1}$ and null space $\mathcal{A}_{2}$. The identity mapping is denoted by $I$. The range and null spaces are denoted by $\mathcal{R}(\cdot)$ and $\mathcal{N}(\cdot)$, respectively. Inner products and norms are denoted by $\langle\mathbf{a}, \mathbf{b}\rangle_{\mathcal{H}}$ and $\|\mathbf{a}\|_{\mathcal{H}}$, with $\mathcal{H}$ being the Hilbert space involved. The norm $\|A\|$ of a linear operator $A$ is its spectral norm.

The Moore-Penrose pseudoinverse [22] and the adjoint of a bounded transformation $T$ are written as $T^{\dagger}$ and $T^{*}$, respectively. If $T_{1}: \mathcal{H}_{1} \rightarrow \mathcal{H}_{2}$ is a linear bounded operator with closed range space, then the Moore-Penrose pseudoinverse $T_{1}^{\dagger}$ exists [22, pp. 321]. If in addition, $T_{2}$ is a linear bijection on $\mathcal{H}_{1}$ then $\mathcal{R}\left(T_{1}\right)=\mathcal{R}\left(T_{1} T_{2}\right)$. Therefore, for a linear and bounded bijection $T_{2}$, and linear, bounded $T_{1}$ with closed range, $\left(T_{1} T_{2}\right)^{\dagger}$ exists.

An easy way to describe linear combinations and inner products is by utilizing set transformations. A set transformation $S: l_{2} \rightarrow \mathcal{H}$ corresponding to frame [23] vectors $\left\{\mathbf{s}_{n}\right\}$ is defined by $S c=\sum_{n} c[n] \mathbf{s}_{n}$ for all $c \in l_{2}$. From the definition of the adjoint, if $c=S^{*} \mathbf{x}$, then $c[n]=\left\langle\mathbf{s}_{n}, \mathbf{x}\right\rangle$. A direct sum $\mathcal{A}_{1} \oplus \mathcal{A}_{2}$ between two closed subspaces $\mathcal{A}_{1}$ and $\mathcal{A}_{2}$ of a Hilbert space $\mathcal{H}$ is the sum set $\mathcal{A}_{1}+\mathcal{A}_{2}=\left\{\mathbf{a}_{1}+\mathbf{a}_{2} ; \mathbf{a}_{1} \in \mathcal{A}_{1}, \mathbf{a}_{2} \in \mathcal{A}_{2}\right\}$ with the property $\mathcal{A}_{1} \cap \mathcal{A}_{2}=\{0\}$. For an operator $T$ and a subspace $\mathcal{A}$, we denote by $T(\mathcal{A})$ the set obtained by applying $T$ to all vectors in $\mathcal{A}$.

We denote by $M: L_{2}(\mathbb{R}) \rightarrow L_{2}(\mathbb{R})$ a nonlinear memoryless (i.e., static) mapping, which maps an input function $\mathbf{y}$ to an output signal $\mathbf{x}=M(\mathbf{y})$. Being static, there is a functional $\mathbf{m}: \mathbb{R} \rightarrow \mathbb{R}$ describing the input-output relation at each time instance $t$, such that $\mathbf{x}(t)=M(\mathbf{y})(t)=\mathbf{m}(\mathbf{y}(t))$ [see Fig. 1(b)]. The derivative of $\mathbf{m}(t)$ is denoted by $\mathbf{m}^{\prime}(t)$. We will also use 
the Fréchet derivative [24] of $M$ to describe $\mathbf{m}^{\prime}(t)$ in terms of an operator in $L_{2}$.

Definition 1: An operator $M: \mathcal{H}_{1} \rightarrow \mathcal{H}_{2}$ is Fréchet differentiable at $\mathbf{y}_{0}$, if there is a linear operator $M_{\mathbf{y}_{0}}^{\prime}: \mathcal{H}_{1} \rightarrow \mathcal{H}_{2}$, such that in a neighborhood of $\mathbf{y}_{0}$

$$
\lim _{\left\|\mathbf{y}-\mathbf{y}_{0}\right\|_{\mathcal{H}_{1}} \rightarrow 0} \frac{\left\|M(\mathbf{y})-M\left(\mathbf{y}_{0}\right)-M_{\mathbf{y}_{0}}^{\prime}\left(\mathbf{y}-\mathbf{y}_{0}\right)\right\|_{\mathcal{H}_{2}}}{\left\|\mathbf{y}-\mathbf{y}_{0}\right\|_{\mathcal{H}_{1}}}=0 .
$$

We refer to $M_{\mathbf{y}_{0}}^{\prime}$ as the Fréchet derivative (or simply the derivative) of $M$ at $\mathbf{y}_{0}$. Note that the Fréchet derivative of a linear operator is the operator itself.

In our setup, $M$ is memoryless, so that the image $\mathbf{x}=M(\mathbf{y})$ is completely determined in terms of the composition $\mathbf{m}(\mathbf{y}(t))=\mathbf{x}(t)$, i.e., $M(\mathbf{y})(t)=\mathbf{m}(\mathbf{y}(t))$. In such a setting, $M_{\mathbf{y}_{0}}^{\prime}$, the Fréchet derivative of $M$ at $\mathbf{y}_{0}$ satisfies $\left(M_{\mathbf{y}_{0}}^{\prime} \mathbf{f}\right)(t)=\mathbf{m}^{\prime}\left(\mathbf{y}_{0}(t)\right) \mathbf{f}(t)$, for any function $\mathbf{f}$, and for all time instances $t$. For example, the Fréchet derivative of the memoryless operator $M(\mathbf{y})(t)=\mathbf{y}^{3}(t)$ evaluated at $\mathbf{y}_{0}$ is determined by the functional $3 \mathbf{y}_{0}^{2}$, and $\left(M_{\mathbf{y}_{0}}^{\prime} \mathbf{f}\right)(t)=3 \mathbf{y}_{0}^{2}(t) \mathbf{f}(t)$.

\section{A. Mathematical Safeguards}

Our main treatment concerns the Hilbert space of real-valued, finite-energy functions. Throughout the paper we assume that the sampling functions $\left\{\mathbf{s}_{n}\right\}$ form a frame [23] for the closure of their span, which we denote by the sampling space $\mathcal{S} \subseteq L_{2}$. Thus, there are constants $0<L_{S} \leq U_{S}<\infty$ such that

$$
L_{S}\|\mathbf{f}\|_{L_{2}} \leq\left\|S^{*} \mathbf{f}\right\|_{l_{2}} \leq U_{S}\|\mathbf{f}\|_{L_{2}}
$$

for all $\mathbf{f} \in \mathcal{S}$, where $S$ is the set transform corresponding to $\left\{\mathbf{s}_{n}\right\}$.

To assure that the inner products $\left\langle\mathbf{s}_{n}, M(\mathbf{y})\right\rangle_{L_{2}}$ are well defined, we assume that the distorted function $\mathbf{x}=M(\mathbf{y})$ is in $L_{2}$ for all $\mathbf{y} \in L_{2}$. The latter requirement is satisfied if, for example, $\mathbf{m}(0)=0$ and $\mathbf{m}$ is Lipschitz continuous. Indeed, in this case

$$
\begin{aligned}
\|M(\mathbf{y})\|_{L_{2}}^{2} & =\int_{-\infty}^{\infty}|\mathbf{m}(\mathbf{y}(t))|^{2} d t \\
& =\int_{-\infty}^{\infty}|\mathbf{m}(\mathbf{y}(t))-\mathbf{m}(0)|^{2} d t \\
& \leq U_{g}^{2} \int_{-\infty}^{\infty}|\mathbf{y}(t)-0|^{2} d t \\
& =U_{g}^{2}\|\mathbf{y}\|_{L_{2}}^{2}<\infty
\end{aligned}
$$

where $U_{g}$ is a Lipschitz bound of $\mathbf{m}$. Another case in which $\mathbf{x}=M(\mathbf{y})$ has finite energy, is when $\mathbf{m}$ is Lipschitz continuous and the input function $\mathbf{y}$ has finite support.

Lipschitz continuity of the nonlinear mapping can be guaranteed by requiring that $\left\|\mathbf{m}^{\prime}\right\|_{L_{\infty}}=\max _{t}\left|\mathbf{m}^{\prime}(t)\right| \leq U_{g}$. Throughout our derivations we also assume that $M$ is invertible. In particular, this holds if the input-output distortion curve $\mathbf{m}$ is a strictly ascending function, ${ }^{1}$ i.e., $\mathbf{m}^{\prime}(t)>0$.

\footnotetext{
${ }^{1}$ The results of this work can be extended for the strictly descending case as well; see Section IX.
}

In summary, our hypothesis is that the slope of the nonlinear distortion satisfies

$$
L_{g} \leq \mathbf{m}^{\prime}(t) \leq U_{g}
$$

for some $0<L_{g}, U_{g}<\infty$, and all $t \in \mathbb{R}$.

To reconstruct functions within some closed subspace $\mathcal{A}$ of $L_{2}$, let $\left\{\mathbf{a}_{n}(t)\right\}$ to be a Riesz basis [23] of $\mathcal{A}$. Then the corresponding set transformation $A$ satisfies

$$
L_{A}\|a\|_{l_{2}} \leq\|A a\|_{L_{2}} \leq U_{A}\|a\|_{l_{2}}
$$

for some fixed $0<L_{A} \leq U_{A}<\infty$, and all $a \in l_{2}$.

\section{PROBLEM Formulation}

Our problem is to reconstruct a continuous-time signal $\mathbf{y}(t)$ from samples of $\mathbf{x}(t)$, which is obtained by a nonlinear, memoryless and invertible mapping $M$ of $\mathbf{y}$, i.e.,

$$
\mathbf{x}=M(\mathbf{y}) .
$$

This nonlinear distortion of amplitudes is illustrated in Fig. 1(b), where a functional $\mathbf{m}$ describes the input-output relation of the nonlinearity. Our measurements are modelled as the generalized samples of $\mathbf{x}=M(\mathbf{y})$, with the $n$th sample given by

$$
c[n]=\left\langle\mathbf{s}_{n}, \mathbf{x}\right\rangle_{L_{2}}=\int_{-\infty}^{\infty} \mathbf{s}_{n}(t) \mathbf{x}(t) d t .
$$

Here, $\mathbf{s}_{n}$ is the $n$th sampling function. We define $\mathcal{S} \subseteq L_{2}$ to be the sampling space, which is the closure of $\operatorname{span}\left\{\mathbf{s}_{n}\right\}$ and $S$ to be the set transformation corresponding to $\left\{\mathbf{s}_{n}\right\}$. With this notation, the generalized samples (6) can be written as

$$
c=S^{*} \mathbf{x} .
$$

By the Riesz representation theorem, the sampling model (7) can describe any linear and bounded sampling scheme.

An important special case of sampling, is when $\mathcal{S}$ is a shift-invariant (SI) subspace, obtained by equidistant shifts of a generator $s(t)$

$$
\mathcal{S}=\left\{f(t): f(t)=\sum_{n} a[n] s(t-n T), a \in l_{2}\right\} .
$$

An example is an ADC which performs prefiltering prior to sampling, as shown in Fig. 2. In such a setting, the sampling vectors $\left\{\mathbf{s}_{n}(t)=\mathbf{s}(t-n T)\right\}$ are shifted and mirrored versions of the prefilter impulse response [9]. Furthermore, the sampling functions form a frame for the closure of their span [23], [25] if and only if

$$
\alpha \leq R_{\mathbf{S}, \mathbf{S}}^{f}(\omega) \leq \beta, \quad \omega \in \mathcal{I}_{\mathcal{S}}
$$

for some $0<\alpha \leq \beta<\infty$. Here we denote,

$$
R_{\mathbf{A}, \mathbf{B}}^{f}(\omega)=\frac{1}{T} \sum_{k=-\infty}^{\infty} \overline{\mathbf{A}^{F}}\left(\frac{\omega+2 \pi k}{T}\right) \mathbf{B}^{F}\left(\frac{\omega+2 \pi k}{T}\right)
$$




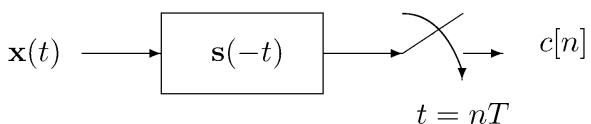

Fig. 2. Filtering with impulse response $\mathbf{s}(-t)$ followed by ideal sampling. The sampling vectors are $\{\mathbf{s}(t-n T)\}$.

where $\mathbf{S}^{F}(\omega)$ is the continuous-time Fourier transform of the generator $\mathbf{s}(t)$, and $\mathcal{I}_{\mathcal{S}}$ is the set of frequencies $\omega$ for which $R_{\mathrm{S}, \mathrm{S}}^{f}(\omega) \neq 0$.

If there are no constraints on $\mathbf{y}$, then the problem of reconstructing this input from the samples $c$ becomes ill-posed. Specifically, there are infinitely many functions of $L_{2}$ which can yield the known samples. Indeed, any signal of the form $M^{-1}\left(\mathbf{x}_{s}+\mathbf{v}\right)$ is a possible candidate, where $\mathbf{v}$ is an arbitrary vector in $\mathcal{S}^{\perp}$ and

$$
\mathbf{x}_{s}=P_{\mathcal{S}} \mathbf{x}=S\left(S^{*} S\right)^{\dagger} c
$$

is the orthogonal projection of $\mathbf{x}$ onto the sampling space, which is uniquely determined by the samples $c$. However, in many practical problems we assume some subspace structure on the input signal. The assumption of a signal being band-limited is probably the most common scenario, though, in this work, we are not limited to the band-limited setup. Our formulation treats the problem of reconstructing $\mathbf{y}$ which is known to lie in an arbitrary closed subspace $\mathcal{A} \subseteq L_{2}$. The overall sampling scheme we consider is illustrated in Fig. 1.

To recover $\mathbf{y}$ from its samples, we need to determine a bijection between this function and the sample sequence. Unfortunately, though we restrict the solution to a closed linear subspace $\mathcal{A}$ of $L_{2}$, it is still possible to have infinitely many functions in $\mathcal{A}$ which can explain the samples. For example, even for a simplified setup of our problem where $M$ is replaced by the identity mapping, there are infinitely many consistent solutions if $\mathcal{A}+\mathcal{S}^{\perp}=L_{2}$ but $\mathcal{A} \cap \mathcal{S}^{\perp} \neq\{0\}$. Indeed, for any $\mathbf{v} \in \mathcal{A} \cap \mathcal{S}^{\perp}$ we have $c=S^{*} \mathbf{y}=S^{*}(\mathbf{y}+\mathbf{v})$. If, however, $\mathcal{A}$ and $\mathcal{S}$ satisfy the direct sum condition

$$
\mathcal{A} \oplus \mathcal{S}^{\perp}=L_{2}
$$

then it is well known [10], [14] that for $M=I$, a unique consistent solution exists. In that case, the consistent solution is also the perfect reconstruction of the true input $\mathbf{y} \in \mathcal{A}$. Thus, if $M=I$ and (10) holds, then the problem is trivial.

In our setup, however, $M \neq I$. Furthermore, $M$ is not even a linear operator. Instead of ignoring the effects of the nonlinearity, yet another simplification of the problem might be to assume that the samples are ideal, i.e., $c[n]=\mathbf{x}(n T)$. In that case, we can resort to Zhu's sampling theorem [8], by applying $M^{-1}$ to the samples $c$. Presuming that indeed $c[n]=\mathbf{x}(n T)$, by this approach we then obtain the ideal samples $\mathbf{y}(n T)$ of $\mathbf{y}$. The problem then reduces to that of recovering a signal $\mathbf{y}$ in a subspace $\mathcal{A}$ from its ideal samples, which has been treated extensively in the sampling literature (see, for example, [26]).

As mentioned, however, in our setup the signal is distorted by a nonlinear mapping $M$, and generalized (rather than ideal) sampling takes place. Hence, approaches which ignore the nonlinearity or the nonideal nature of the sampling scheme are sub- optimal, and in general, will not lead to perfect recovery of the input y. In Section X, we demonstrate that by applying $M^{-1}$ directly to the samples and then recovering $\mathbf{y}$ leads to suboptimal reconstruction performance. Nonetheless, we will show that if (10) is satisfied, and under proper assumptions on the nonlinear distortion $M$, then there is a unique function $\mathbf{y}$ within $\mathcal{A}$, which can explain the measured samples. Building on this result we develop the theory and a concrete iterative method for obtaining perfect reconstruction of a signal in a subspace, despite the fact that it is measured through a nonlinear and nonideal acquisition device.

Before treating this general case, we note that there are special setups for which it is possible to reconstruct the function $\mathbf{y}$ in a closed form. An example of such a setup is presented in the following theorem.

Theorem 1: Let $\mathbf{y}(t)$ be a periodic function with period $T_{0}=$ $2 \pi / \omega_{0}$ that satisfies the Dirichlet conditions. Let $M$ be a Lipschitz continuous, memoryless and invertible mapping. If $\mathbf{x}=$ $M(\mathbf{y})$ is sampled with the sampling functions

$$
\mathbf{s}_{n}(t)=e^{-j \omega_{0} n t} \mathbf{s}(t)
$$

and the generator $\mathbf{s}(t)$ satisfies

$$
0<\left|\sum_{k} \mathbf{s}\left(t-T_{0} k\right)\right|<\infty
$$

for all $t \in\left[0, T_{0}\right]$, then $\mathbf{y}(t)$ can be reconstructed from the generalized samples (6). The reconstruction is given by

$$
\mathbf{y}(t)=M^{-1}\left(\sum_{m}\left(c * h^{-1}\right)[m] e^{j \omega_{0} m t}\right)
$$

where $h^{-1}$ is the convolutional inverse of $h[n]=\mathbf{S}^{F}\left(\omega_{0} n\right)$, and $\mathbf{S}^{F}$ is the continuous-time Fourier transform of $\mathbf{s}$.

Proof: See Appendix I.

In Theorem 1, a special choice of sampling functions is employed in order to reconstruct a periodic function. In the general case, however, the sampling functions do not satisfy (11). Hence, the rest of this paper will treat a much broader setting, allowing the use of arbitrary sampling and reconstruction spaces. In particular, the more standard setup of SI spaces is included in our framework.

\section{UNIQUENESS}

In this section, we prove that under proper conditions on $M$ and the spaces involved, the problem of perfectly reconstructing the input signal from its nonlinear and generalized samples indeed has a unique solution. Specifically, we show that if the subspace $M_{\mathbf{h}}^{\prime}(\mathcal{A})$ (i.e., the space obtained by applying the Fréchet derivative $M_{\mathrm{h}}^{\prime}$ to each vector in $\mathcal{A}$ ) satisfies the direct $\operatorname{sum} M_{\mathbf{h}}^{\prime}(\mathcal{A}) \oplus \mathcal{S}^{\perp}=L_{2}$ for all $\mathbf{h} \in L_{2}$, then $\mathbf{y} \in \mathcal{A}$ is uniquely determined by its samples.

Theorem 2: Assume $M_{\mathbf{h}}^{\prime}(\mathcal{A}) \oplus \mathcal{S}^{\perp}=L_{2}$ for all $\mathbf{h} \in L_{2}$. Then there is a unique $\mathbf{y} \in \mathcal{A}$ such that $c=S^{*} M(\mathbf{y})$.

Proof: Assume that there are two functions $\mathbf{y}_{1} \in \mathcal{A}, \mathbf{y}_{2} \in \mathcal{A}$ which both satisfy $c=S^{*} M\left(\mathbf{y}_{1}\right)=S^{*} M\left(\mathbf{y}_{2}\right)$. Then

$$
P_{\mathcal{S}} M\left(\mathbf{y}_{1}\right)=P_{\mathcal{S}} M\left(\mathbf{y}_{2}\right)=\mathbf{x}_{s},
$$


implying that $M\left(\mathbf{y}_{1}\right)-M\left(\mathbf{y}_{2}\right) \in \mathcal{S}^{\perp}$.

For each time instance $t$, we have some interval of amplitudes $\left[\mathbf{y}_{1}(t), \mathbf{y}_{2}(t)\right]$, where we have assumed, without loss of generality, that $\mathbf{y}_{1}(t) \leq \mathbf{y}_{2}(t)$. Since by (4) the nonlinear distortion curve $\mathbf{m}$ is continuous and differentiable, by the mean value theorem, there is a scalar $\alpha_{t} \in[0,1]$ and an intermediate value $h_{t}=\alpha_{t} \mathbf{y}_{1}(t)+\left(1-\alpha_{t}\right) \mathbf{y}_{2}(t)$ such that

$$
\mathbf{m}^{\prime}\left(h_{t}\right)\left(\mathbf{y}_{1}(t)-\mathbf{y}_{2}(t)\right)=\mathbf{m}\left(\mathbf{y}_{1}(t)\right)-\mathbf{m}\left(\mathbf{y}_{2}(t)\right) .
$$

Defining a function $\mathbf{h}(t)=h_{t}$, we may rewrite (14) for all $t$ using operator notations

$$
M_{\mathbf{h}}^{\prime}\left(\mathbf{y}_{1}-\mathbf{y}_{2}\right)=M\left(\mathbf{y}_{1}\right)-M\left(\mathbf{y}_{2}\right) .
$$

The resulting function $M_{\mathbf{h}}^{\prime}\left(\mathbf{y}_{1}-\mathbf{y}_{2}\right)$ lies within the subspace $M_{\mathrm{h}}^{\prime}(\mathcal{A})$ and by the right-hand side of (15) it is also a function in $\mathcal{S}^{\perp}$. By the direct sum assumption $M_{\mathbf{h}}^{\prime}(\mathcal{A}) \oplus \mathcal{S}^{\perp}=L_{2}$, we must have $M\left(\mathbf{y}_{1}\right)-M\left(\mathbf{y}_{2}\right)=0$, or equivalently (since $M$ is a bijection), $\mathbf{y}_{1}=\mathbf{y}_{2}$.

In the sequel we will state simple conditions on $M$ and the spaces $\mathcal{A}$ and $\mathcal{S}$, which assure that the direct sum assumptions of Theorem 2 are met in practice. In particular, we will show that if $M$ is smooth enough, then (10) is sufficient to ensure uniqueness.

\section{RESTORATION VIA LINEARIZATION}

Under the conditions of Theorem 2, there is a unique function $\mathbf{y} \in \mathcal{A}$ which is consistent with the measured samples $c$. Therefore, all we need is to find $\mathbf{y} \in \mathcal{A}$ satisfying $c=S^{*} M(\mathbf{y})$. A natural approach to retrieve $\mathbf{y}$ is to iteratively linearize these nonlinear equations. As this method relies on linearization, we first need to explain in detail, how to recover the input $\mathbf{y} \in \mathcal{A}$ if it is related to $\mathbf{x}$ through a linear mapping.

\section{A. Perfect Reconstruction for Linear Schemes}

In this section we adopt a general formulation within some Hilbert space $\mathcal{H}$ (which is not necessarily $L_{2}$ ). Assume a model where $\mathbf{y}$ is related to $\mathbf{x}$ through a linear, bounded and bijective mapping $T$, i.e.,

$$
\mathbf{x}=T \mathbf{y}
$$

The case $T=I$ was previously addressed in the literature [9], [10], [13], [14]. Assuming that the direct sum condition (10) is satisfied, it is known that $\mathbf{y} \in \mathcal{A}$ can be perfectly reconstructed from the samples of $\mathbf{x}$, by oblique projecting $\mathbf{x}_{s}$ of (9) along $\mathcal{S}^{\perp}$ onto the reconstruction space $\mathcal{A}$ :

$$
\mathbf{y}=P_{\mathcal{A}, \mathcal{S} \perp} \mathbf{x}_{s}
$$

The extension of this result to any linear continuous and continuously invertible $T$ (not necessarily $T=I$ ) is simple. First note that since the solution $\mathrm{y}$ lies within $\mathcal{A}$, the function $\mathrm{x}$ is constrained to the subspace $T(\mathcal{A})=\{T \mathbf{y}: \mathbf{y} \in \mathcal{A}\}$. Furthermore, in our context $T$ will play the role of the operator $M_{\mathbf{h}}^{\prime}$ of

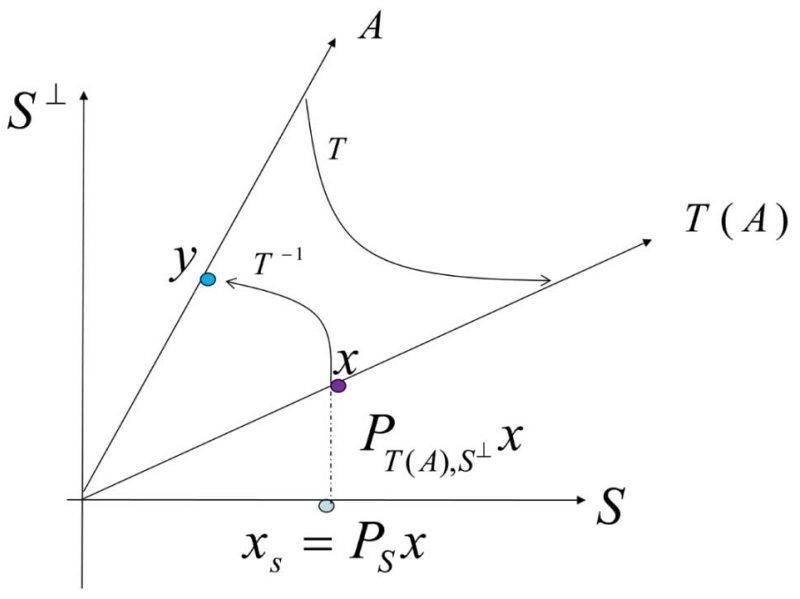

Fig. 3. Perfect reconstruction with linear invertible mapping.

Theorem 2, and we will derive conditions to assure that the direct sum $T(\mathcal{A}) \oplus \mathcal{S}^{\perp}=\mathcal{H}$ holds. Then, perfect reconstruction of any $\mathrm{x} \in T(\mathcal{A})$ is given by

$$
\mathbf{x}=P_{T(\mathcal{A}), \mathcal{S}^{\perp}} P_{\mathcal{S}} \mathbf{x}=P_{T(\mathcal{A}), \mathcal{S}} \mathbf{x}_{s} .
$$

Also note that due to the direct sum assumption $T(\mathcal{A}) \oplus \mathcal{S}^{\perp}=$ $\mathcal{H}$, the oblique projection operator $P_{T(\mathcal{A}), \mathcal{S}^{\perp}}$ is well defined (e.g., [25]). Finally, to obtain y itself, we apply $T^{-1}$ to (17). This simple idea is illustrated in Fig. 3 and summarized in the following theorem.

Theorem 3: Let $\mathbf{y} \in \mathcal{A} \subseteq \mathcal{H}$ and let $\mathbf{x}=T \mathbf{y}$ with $T$ : $\mathcal{H} \rightarrow \mathcal{H}$ a linear, continuous and bijective mapping satisfying $T(\mathcal{A}) \oplus \mathcal{S}^{\perp}=\mathcal{H}$. Then we can reconstruct $\mathbf{y}$ from the samples $c=S^{*} \mathbf{x}$ by

$$
\mathbf{y}=T^{-1} P_{T(\mathcal{A}), \mathcal{S}^{\perp}} \mathbf{X}_{s}=A\left(S^{*} T A\right)^{\dagger} c
$$

where $A$ is a set transformation corresponding to a Riesz basis for $\mathcal{A}$

In (18) we have used $P_{T(\mathcal{A}), \mathcal{S}^{\perp}} \mathbf{x}_{s}=T A\left(S^{*} T A\right)^{\dagger} c$. For the special case $T=I$ we obtain the expected oblique projection solution [9], [13], [14] of (16).

\section{B. Iterating Oblique Projections}

We now use the results on the linear case to develop an iterative recovery algorithm in the presence of nonlinearities. The true input $\mathbf{y}$ is a function consistent with the measured samples $c$, i.e., it satisfies

$$
c=S^{*} M(\mathbf{y})
$$

To recover y we may first linearize (19) by starting with some initial guess $\mathbf{y}_{0} \in \mathcal{A}$ and approximating the memoryless nonlinear operator $M$ using its Fréchet derivative at $\mathbf{y}_{0}$

$$
M(\mathbf{y}) \approx M\left(\mathbf{y}_{0}\right)+M_{0}^{\prime}\left(\mathbf{y}-\mathbf{y}_{0}\right)
$$




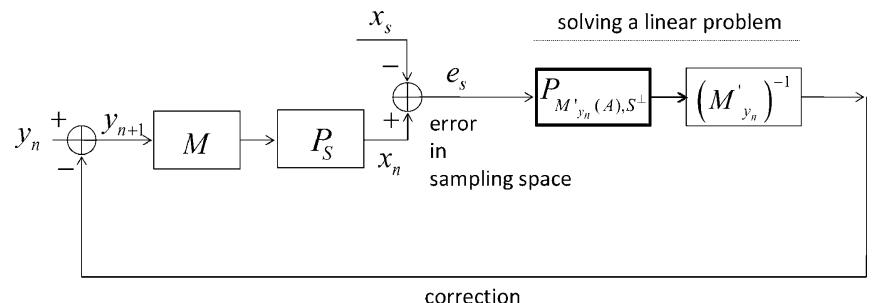

Fig. 4. Schematic of the iterative algorithm.

where for brevity we denoted $M_{n}^{\prime}=M_{\mathbf{y}_{n}}^{\prime}$. Rewriting (19) using (20) yields

$$
S^{*} M_{0}^{\prime} \mathbf{y}=c-S^{*}\left(M\left(\mathbf{y}_{0}\right)-M_{0}^{\prime} \mathbf{y}_{0}\right) .
$$

The left-hand side of (21) describes a vector $M_{0}^{\prime} \mathbf{y}$ which lies within the subspace $M_{0}^{\prime}(\mathcal{A})$ and is sampled by the analysis operator $S^{*}$. The right hand is the resulting sample sequence. Since by (4) the linear derivative operator $M_{0}^{\prime}$ is a bounded bijection on $L_{2}$, we may apply the result of Theorem 3 as long as the direct sum condition $M_{0}^{\prime}(\mathcal{A}) \oplus \mathcal{S}^{\perp}=L_{2}$ is satisfied. Specifically, identifying $M_{0}^{\prime}$ with $T$ in Theorem 3 , the unique solution of (21) is

$$
\begin{aligned}
\hat{\mathbf{y}} & =\left(M_{0}^{\prime}\right)^{-1} P_{M_{0}^{\prime}(\mathcal{A}), \mathcal{S}^{\perp}}\left(\mathbf{x}_{s}-\left(M\left(\mathbf{y}_{0}\right)-M_{0}^{\prime} \mathbf{y}_{0}\right)\right) \\
& =\mathbf{y}_{0}-\left(M_{0}^{\prime}\right)^{-1} P_{M_{0}^{\prime}(\mathcal{A}), \mathcal{S}^{\perp}}\left(M\left(\mathbf{y}_{0}\right)-\mathbf{x}_{s}\right)
\end{aligned}
$$

where $\mathbf{x}_{s}=P_{\mathcal{S}} \mathbf{x}$ such that $c=S^{*} \mathbf{x}_{s}$. The process can now be repeated, using $\mathbf{y}_{1}=\hat{\mathbf{y}}$ as the new approximation point.

Assuming that for each iteration $n$ we have $M_{n}^{\prime}(\mathcal{A}) \oplus \mathcal{S}^{\perp}=$ $L_{2}$, we may summarize the basic form of the algorithm by

$$
\begin{aligned}
\mathbf{y}_{n+1} & =\mathbf{y}_{n}-\left(M_{n}^{\prime}\right)^{-1} P_{M_{n}^{\prime}(\mathcal{A}), \mathcal{S}^{\perp}}\left(M\left(\mathbf{y}_{n}\right)-\mathbf{x}_{s}\right) \\
& =\mathbf{y}_{n}-\left(M_{n}^{\prime}\right)^{-1} P_{M_{n}^{\prime}(\mathcal{A}), \mathcal{S}^{\perp}}\left(P_{\mathcal{S}} M\left(\mathbf{y}_{n}\right)-\mathbf{x}_{s}\right)
\end{aligned}
$$

where we used $P_{M_{n}^{\prime}(\mathcal{A}), \mathcal{S}^{\perp}}=P_{M_{n}^{\prime}(\mathcal{A}), \mathcal{S}^{\perp}} P_{\mathcal{S}}$ in the last equality. This leads to the following interpretation of the algorithm:

- Using the current approximation $\mathbf{y}_{n}$, calculate $\mathbf{e}_{s}=$ $P_{\mathcal{S}} M\left(\mathbf{y}_{n}\right)-\mathbf{x}_{s}$, the error within the sampling space.

- Solve a linear problem of finding a function within the reconstruction space $\mathcal{A}$, consistent with $\mathbf{e}_{s}$ when $M=M_{n}^{\prime}$. The solution is $\left(M_{n}^{\prime}\right)^{-1} P_{M_{n}^{\prime}(\mathcal{A}), \mathcal{S}^{\perp}} \mathbf{e}_{s}$.

- Update the current estimate $\mathbf{y}_{n}$ using the resulting correction term.

This idea is described schematically in Fig. 4.

Finally, note that in practice, we need only to update the representation coefficients of $\mathbf{y}_{n}$ within $\mathcal{A}$. Thus, we may write $\mathbf{y}_{n}=A a_{n}$, where the set transformation $A$ corresponds to a Riesz basis for $\mathcal{A}$, and $a_{n} \in l_{2}$ are the coefficients. This results in a discrete version of the algorithm (23):

$$
a_{n+1}=a_{n}-\left(S^{*} M_{n}^{\prime} A\right)^{\dagger}\left(S^{*} M\left(A a_{n}\right)-c\right)
$$

where we used $\left(M_{n}^{\prime}\right)^{-1} P_{M^{\prime}(\mathcal{A}) \mathcal{S}^{\perp}}=\left(S^{*} M_{n}^{\prime} A\right)^{\dagger} S^{*}$, and $S^{*} \mathbf{x}_{s}=c$. Note that the pseudoinverse is well defined due to the direct sum assumption $M_{n}^{\prime}(\mathcal{A}) \oplus \mathcal{S}^{\perp}=L_{2}$ [25].

\section{The POCS PoINT OF VIEW}

A different approach for tackling our problem can be obtained by the POCS algorithm [17]. In this section we will show the equivalence between the POCS method and the iterative oblique projections (23)

First note that the unknown input signal lies in the intersection of two sets: The subspace $\mathcal{A}$ and the set

$$
\mathcal{U}=\left\{\mathbf{y}: S^{*} M(\mathbf{y})=c\right\}
$$

of all functions which can yield the known samples. For nonlinear $M$, the set $\mathcal{U}$ of (25) is in general nonconvex. POCS methods are successfully used even in problems where we iterate projections between a convex set and a nonconvex one, assuming that it is known how to compute the projections onto the sets involved (e.g., [27] and [28]). Unfortunately, in our problem, it is hard to compute the orthogonal projection onto $\mathcal{U}$. However, we can approximate $\mathcal{U}$ using an affine (and hence convex) subset. Replacing the operator $M$ with its linearization around some estimate $\mathbf{y}_{n}$, i.e., $M(\mathbf{y}) \approx M\left(\mathbf{y}_{n}\right)+M_{n}^{\prime}\left(\mathbf{y}-\mathbf{y}_{n}\right)$, allows us to locally approximate $\mathcal{U}$ by the set

$$
\Omega_{n}=\left\{\mathbf{y}: S^{*} M_{n}^{\prime} \mathbf{y}=r_{n}\right\}
$$

where we define

$$
r_{n}=S^{*} M_{n}^{\prime} \mathbf{y}_{n}-S^{*} M\left(\mathbf{y}_{n}\right)+c .
$$

Note that when $M$ is linear, $r_{n}=c$. For nonlinear $M, r_{n}$ also contains a residual term due to approximating $M$ by its Fréchet derivative.

We point out that the set $\Omega_{n}$ is never empty; indeed, $r_{n} \in$ $\mathcal{R}\left(S^{*}\right)$, but since by (4) $M_{n}^{\prime}$ is bijective, then $\mathcal{R}\left(S^{*} M_{n}^{\prime}\right)=$ $\mathcal{R}\left(S^{*}\right)$. In addition, since $M_{n}^{\prime}$ is also bounded, the Moore-Penrose pseudoinverse of $S^{*} M_{n}^{\prime}$ is well defined (see Section II). Therefore, we may rewrite $\Omega_{n}$ as the affine subset

$$
\Omega_{n}=\left(S^{*} M_{n}^{\prime}\right)^{\dagger} r_{n}+\mathcal{N}\left(S^{*} M_{n}^{\prime}\right) .
$$

Given a vector $\mathbf{z}$, its projection onto $\Omega_{n}$ is given by

$$
P_{\Omega_{n}} \mathbf{z}=\left(S^{*} M_{n}^{\prime}\right)^{\dagger} r_{n}+P_{\mathcal{N}\left(S^{*} M_{n}^{\prime}\right)} \mathbf{z} .
$$

Using (29), we now apply the POCS algorithm to this approximated problem of finding an element within the intersection $\mathcal{A} \cap \Omega_{n}$.

Starting with some initialization $\mathbf{h}_{n}^{0}$, the POCS iterations take the form

$$
\mathbf{h}_{n}^{m+1}=P_{\Omega_{n}} P_{\mathcal{A}} \mathbf{h}_{n}^{m}
$$

where $n$ is held fixed, and $m$ is the iteration index. As long as the intersection $\mathcal{A} \cap \Omega_{n}$ is non empty, for any initialization $\mathbf{h}_{n}^{0}$, the iterations (30) are known to converge [17] to some element within $\mathcal{A} \cap \Omega_{n}$. Once convergence has been obtained, the process 
can be repeated by setting a new linearization point $\mathbf{y}_{n+1}=\mathbf{h}_{n}^{\infty}$ and defining a new approximation set, $\Omega_{n+1}$, similarly to (28). Combining (30) with (29) we have $\mathbf{h}_{n}^{m+1}=\left(S^{*} M_{n}^{\prime}\right)^{\dagger} r_{n}+$ $P_{\mathcal{N}\left(S^{*} M_{n}^{\prime}\right)} P_{\mathcal{A}} \mathbf{h}_{n}^{m}$. Continuing this expansion, while expressing the result in terms of the initial guess $\mathbf{h}_{n}^{0}$, leads to

$$
\begin{aligned}
\mathbf{h}_{n}^{m+1}= & \sum_{i=0}^{m}\left(P_{\mathcal{N}\left(S^{*} M_{n}^{\prime}\right)} P_{\mathcal{A}}\right)^{i}\left(S^{*} M_{n}^{\prime}\right)^{\dagger} r_{n} \\
& +\left(P_{\mathcal{N}\left(S^{*} M_{n}^{\prime}\right)} P_{\mathcal{A}}\right)^{m+1} \mathbf{h}_{n}^{0} \quad \forall m \geq 0 .
\end{aligned}
$$

Substituting $\mathbf{y}_{n+1}=\mathbf{h}_{n}^{\infty}$, in the limit we have

$$
\begin{aligned}
\mathbf{y}_{n+1}=\sum_{i=0}^{\infty}\left(P_{\mathcal{N}\left(S^{*} M_{n}^{\prime}\right)} P_{\mathcal{A}}\right)^{i} & \left.S^{*} M_{n}^{\prime}\right)^{\dagger} r_{n} \\
& +\left(P_{\mathcal{N}\left(S^{*} M_{n}^{\prime}\right)} P_{\mathcal{A}}\right)^{\infty} \mathbf{h}_{n}^{0} .
\end{aligned}
$$

Interestingly, the infinite sum (31), can be significantly simplified if again we assume that the direct sum conditions

$$
M_{n}^{\prime}(\mathcal{A}) \oplus \mathcal{S}^{\perp}=L_{2}
$$

are satisfied for all $n$. In fact, the POCS method becomes equivalent to the approach presented in the previous section.

Theorem 4: Assume $M_{n}^{\prime}(\mathcal{A}) \oplus \mathcal{S}^{\perp}=L_{2}$ holds for all $n$. Then iterations (31) are equivalent to (23).

Proof: See Appendix II.

\section{LinEARIZATION AS FRAME PERTURbation TheORY}

We have seen that under the direct sum conditions (32) both the iterative oblique projections and the approximated POCS method take on the form (23). Furthermore, as stated in Theorem 2, such direct sum conditions are also vital to prove uniqueness of the solution. Ensuring that for any linearization point $\mathbf{y}_{n}$, the direct sum $M_{n}^{\prime}(\mathcal{A}) \oplus \mathcal{S}^{\perp}$ holds is not trivial. In this section we derive sufficient (and simple to verify) conditions on the nonlinear distortion $M$, which assure this condition.

The key idea is to view the linearization process, which led to the modified subspace $M_{n}^{\prime}(\mathcal{A})$, as a perturbation of the original space $\mathcal{A}$. If the perturbation is 'small enough', and $\mathcal{A} \oplus \mathcal{S}^{\perp}=$ $L_{2}$, then we can prove (32). Before proceeding with the mathematical derivations, it is beneficial to geometrically interpret this idea for $\mathcal{H}=\mathbb{R}^{2}$, as illustrated in Fig. 5. As long as $\mathcal{A} \oplus \mathcal{S}^{\perp}=\mathcal{H}$ holds (here, the line defined by the subspace $\mathcal{A}$ is not perpendicular to $\mathcal{S}$ ) and $M_{n}^{\prime}(\mathcal{A})$ is sufficiently close to $\mathcal{A}$, we can also guarantee that $M_{n}^{\prime}(\mathcal{A}) \oplus \mathcal{S}^{\perp}=\mathcal{H}$, since the angle between $\mathcal{S}$ and the perturbed subspace $M_{n}^{\prime}(\mathcal{A})$ is smaller than $90^{\circ}$.

As shown in Fig. 5, the concept of an angle between spaces is simple for $\mathcal{H}=\mathbb{R}^{2}$. The natural extension of this idea in arbitrary Hilbert spaces is given by the definition of the cosine and sine between two subspaces $\mathcal{A}_{1}, \mathcal{A}_{2}$ of some Hilbert space $\mathcal{H}$ [9], [16]:

$$
\begin{aligned}
& \cos \left(\mathcal{A}_{1}, \mathcal{A}_{2}\right)=\inf _{f \in \mathcal{A}_{1},\|f\|_{\mathcal{H}}=1}\left\|P_{\mathcal{A}_{2}} f\right\|_{\mathcal{H}} \\
& \sin \left(\mathcal{A}_{1}, \mathcal{A}_{2}\right)=\sup _{f \in \mathcal{A}_{1},\|f\|_{\mathcal{H}}=1}\left\|P_{\mathcal{A}_{2}^{\perp}} f\right\|_{\mathcal{H}} .
\end{aligned}
$$

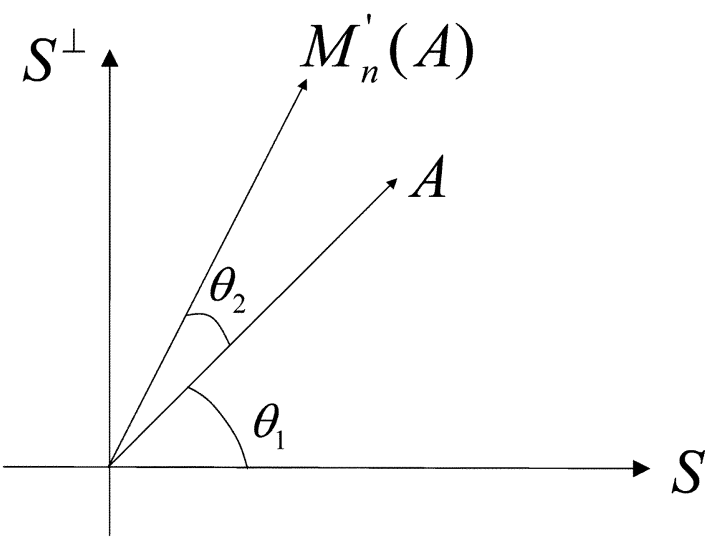

Fig. 5. Subspace $\mathcal{A}$ and its perturbation. As long as the sum of maximal angles between $\mathcal{S}$ and $\mathcal{A}$, and $\mathcal{A}$ with its perturbation $M_{n}^{\prime}(\mathcal{A})$ (i.e., $\theta_{1}$ and $\theta_{2}$, respectively) is smaller than $90^{\circ}$, the direct sum (32) is satisfied.

Throughout this section we will also use the relations [16]

$$
\begin{aligned}
\sin \left(\mathcal{A}_{1}, \mathcal{A}_{2}\right) & =\sin \left(\mathcal{A}_{2}^{\perp}, \mathcal{A}_{1}^{\perp}\right) \\
\cos \left(\mathcal{A}_{1}, \mathcal{A}_{2}\right) & =\cos \left(\mathcal{A}_{2}^{\perp}, \mathcal{A}_{1}^{\perp}\right) \\
\sin ^{2}\left(\mathcal{A}_{1}, \mathcal{A}_{2}\right) & =1-\cos ^{2}\left(\mathcal{A}_{1}, \mathcal{A}_{2}\right) .
\end{aligned}
$$

We start by showing that $M_{n}^{\prime}(\mathcal{A})$ is 'sufficiently close' to $\mathcal{A}$ (in terms of Fig. 5, the angle $\theta_{2}$ is smaller than 90 degrees), by stating a sufficient condition on the nonlinear distortion which guarantees that

$$
\mathcal{A}^{\perp} \oplus M_{n}^{\prime}(\mathcal{A})=L_{2}
$$

along the iterations. We then use (35), to derive sufficient conditions for the direct sum $M_{n}^{\prime}(\mathcal{A}) \oplus \mathcal{S}^{\perp}=L_{2}$ to hold. In terms of Fig. 5, the latter means that $\theta_{1}+\theta_{2}$ is also smaller than 90 degrees.

To show (35), we rely on recent results concerning frame perturbations on a subspace, and extend these to our setup.

Proposition 1: If $\left\|1-\mathbf{m}_{n}^{\prime}(t)\right\|_{L_{\infty}}<1$, then $\mathcal{A}^{\perp} \oplus M_{n}^{\prime}(\mathcal{A})=$ $L_{2}$.

Proof: The direct sum condition $\mathcal{A}^{\perp} \oplus M_{n}^{\prime}(\mathcal{A})=L_{2}$ is equivalent to $\cos \left(\mathcal{A}, M_{n}^{\prime}(\mathcal{A})\right)>0$ and $\cos \left(M_{n}^{\prime}(\mathcal{A}), \mathcal{A}\right)>0$ [16]. The requirement $\cos \left(\mathcal{A}, M_{n}^{\prime}(\mathcal{A})\right)>0$ can be guaranteed by relying on the following lemma.

Lemma 1 [20, Theorem 2.1]: Let $\left\{\mathbf{f}_{k}\right\}$ be a frame for $\mathcal{W} \subset$ $\mathcal{H}$, with frame bounds $A \leq B$. For a sequence $\left\{\tilde{\mathbf{f}}_{k}\right\}$ in $\mathcal{H}$, let $\mathcal{V}:=\overline{\operatorname{span}}\left\{\tilde{\mathbf{f}}_{k}\right\}$, and assume that there exists a constant $\mu>0$ such that

$$
\left\|\sum_{k} c_{k}\left(\mathbf{f}_{k}-\tilde{\mathbf{f}}_{k}\right)\right\|_{\mathcal{H}} \leq \mu\left(\sum_{k}\left|c_{k}\right|^{2}\right)^{1 / 2}
$$

for all finite scalar sequences $\left\{c_{k}\right\}$. Then the following holds:

a) $\left\{\tilde{\mathbf{f}}_{k}\right\}$ is a Bessel sequence ${ }^{2}$ with bound $B\left(\sin \left(\mathcal{V}, \mathcal{W}^{\perp}\right)+\right.$ $\mu / \sqrt{B})^{2}$

b) if $\mu<\sqrt{A}$, then

$\cos (\mathcal{W}, \mathcal{V}) \geq(\sqrt{A}-\mu) /\left(\sqrt{B} \sin \left(\mathcal{V}, \mathcal{W}^{\perp}\right)+\mu\right)>0$.

${ }^{2}\left\{\mathbf{s}_{k}\right\}$ is a Bessel sequence if the right-hand side inequality of (2) is satisfied. 
If, in addition to $b), \cos (\mathcal{V}, \mathcal{W})>0$, then

c) $\left\{\tilde{\mathbf{f}}_{k}\right\}$ is a frame for $\mathcal{V}$ with bounds $A(1-\mu / \sqrt{A})^{2}$, $B\left(\sin \left(\mathcal{V}, \mathcal{W}^{\perp}\right)+\mu / \sqrt{B}\right)^{2}$

d) $\mathcal{V}$ is isomorphic to $\mathcal{W}$.

Substituting $\mathcal{A}$ for $\mathcal{W}, M_{n}^{\prime}(\mathcal{A})$ for $\mathcal{V}$ and $\left\{\tilde{\mathbf{f}}_{k}=M_{n}^{\prime}\left(\mathbf{f}_{k}\right)\right\}$, where we take $\left\{\mathbf{f}_{k}\right\}$ to be an orthonormal basis of $\mathcal{A}$ with set transformation $F$, we can rewrite condition (36) using operator notations as

$$
\left\|\left(I-M_{n}^{\prime}\right) F c\right\|_{\mathcal{H}}<\mu\|c\|_{l_{2}} .
$$

Now

$$
\begin{aligned}
\left\|\left(I-M_{n}^{\prime}\right) F c\right\|_{L_{2}} & \leq \max _{t}\left|1-\mathbf{m}_{n}^{\prime}(t)\right|\|F c\|_{L_{2}} \\
& =\max _{t}\left|1-\mathbf{m}_{n}^{\prime}(t)\right|\|c\|_{l_{2}}
\end{aligned}
$$

where we used the orthonormality of $\left\{\mathbf{f}_{k}\right\}$ in the last equality. Thus, (37) holds with $\mu=\left\|1-\mathbf{m}_{n}^{\prime}\right\|_{L_{\infty}}$. By Lemma 1, part b), this means that as long as

$$
\left\|1-\mathbf{m}_{n}^{\prime}\right\|_{L_{\infty}}<1
$$

then

$$
\cos \left(A, M_{n}^{\prime}(\mathcal{A})\right) \geq \frac{1-\left\|1-\mathbf{m}_{n}^{\prime}\right\|_{L_{\infty}}}{\sin \left(M_{n}^{\prime}(\mathcal{A}), \mathcal{A}^{\perp}\right)+\left\|1-\mathbf{m}_{n}^{\prime}\right\|_{L_{\infty}}}>0 .
$$

To avoid expressions which require the computation of $\sin \left(M_{n}^{\prime}(\mathcal{A}), \mathcal{A}^{\perp}\right)$, we can further lower bound (39) by

$$
\cos \left(A, M_{n}^{\prime}(\mathcal{A})\right) \geq \frac{1-\left\|1-\mathbf{m}_{n}^{\prime}\right\|_{L_{\infty}}}{1+\left\|1-\mathbf{m}_{n}^{\prime}\right\|_{L_{\infty}}}>0
$$

To also establish $\cos \left(M_{n}^{\prime}(\mathcal{A}), \mathcal{A}\right)>0$ we now extend Lemma 1 , by exploiting the special relation between $\mathcal{A}$ and $M_{n}^{\prime}(\mathcal{A})$ (i.e., connected by an invertible, bounded and self adjoint mapping).

Lemma 2: Assume $\cos \left(\mathcal{A}, M_{n}^{\prime}(\mathcal{A})\right)>0$ with $M_{n}^{\prime}$ a bounded, invertible and self adjoint mapping. Then $\cos \left(M_{n}^{\prime}(\mathcal{A}), \mathcal{A}\right)>0$.

Proof: Let $\mathbf{f}=M_{n}^{\prime} \mathbf{a}$ for some $\mathbf{a} \in \mathcal{A}$, such that $\|\mathbf{f}\|_{L_{2}}=1$. Take $A$ to be a set transformation of a Riesz basis of $\mathcal{A}$, with Riesz bounds $L_{A}, U_{A}$ as in (5). Since $\left\|P_{\mathcal{A}} \mathbf{f}\right\|_{L_{2}} \geq$ $L_{A}\left\|\left(A^{*} A\right)^{-1} A^{*} M_{n}^{\prime} \mathbf{a}\right\|_{l_{2}} \geq\left(L_{A} / U_{A}^{2}\right)\left\|A^{*} M_{n}^{\prime} \mathbf{a}\right\|_{l_{2}}$ it is sufficient to show that

$$
\inf _{\left\|M_{n}^{\prime} \mathbf{a}\right\|_{L_{2}}=1, \mathbf{a} \in \mathcal{A}}\left\|A^{*} M_{n}^{\prime} \mathbf{a}\right\|_{l_{2}}>0 .
$$

Exploiting the fact that $M_{n}^{\prime}$ is bijective and self adjoint, we may write

$$
\begin{array}{r}
\cos \left(\mathcal{A}, M_{n}^{\prime}(\mathcal{A})\right) \\
=\inf _{\|\mathbf{a}\|_{L_{2}}=1, \mathbf{a} \in \mathcal{A}}\left\|M_{n}^{\prime} A\left(A^{*} M_{n}^{\prime 2} A\right)^{-1} A^{*} M_{n}^{\prime} \mathbf{a}\right\|_{L_{2}} .
\end{array}
$$

Since $\cos \left(\mathcal{A}, M_{n}^{\prime}(\mathcal{A})\right)>0$ and the operator $M_{n}^{\prime} A\left(A^{*} M_{n}^{\prime 2} A\right)^{-1}$ is bounded, we must have that $\left\|A^{*} M_{n}^{\prime} \mathbf{a}\right\|_{l_{2}} \geq \alpha$ for every unit norm $\mathbf{a}$ in $\mathcal{A}$, and some strictly positive $\alpha$. Thus, the left-hand side of (41) can be lower bounded by

$$
\begin{aligned}
\inf _{\left\|M_{n}^{\prime} \mathbf{a}\right\|_{L_{2}}=1, \mathbf{a} \in \mathcal{A}}\left\|A^{*} M_{n}^{\prime} \mathbf{a}\right\|_{l_{2}} & \geq \inf _{\left\|M_{n}^{\prime} \mathbf{a}\right\|_{L_{2}}=1, \mathbf{a} \in \mathcal{A}} \alpha\|\mathbf{a}\|_{L_{2}} \\
& \geq \frac{\alpha}{U_{g}}>0
\end{aligned}
$$

where we used $1=\left\|M_{n}^{\prime} \mathbf{a}\right\|_{L_{2}} \leq\|\mathbf{a}\|_{L_{2}} U_{g}$ in the last inequality.

Having established that $\cos \left(M_{n}^{\prime}(\mathcal{A}), \mathcal{A}\right)>0$ and $\cos \left(\mathcal{A}, M_{n}^{\prime}(\mathcal{A})\right)>0$, it follows from [16] that $M_{n}^{\prime}(\mathcal{A}) \oplus \mathcal{A}^{\perp}=$ $L_{2}$, completing the proof.

We now show that if $\mathcal{A}$ is 'sufficiently close' to $\mathcal{S}$ then there are nonlinear distortions for which (32) holds.

Theorem 5: Assume $\mathcal{A} \oplus \mathcal{S}^{\perp}=L_{2}$. If

$$
\left\|1-\mathbf{m}_{n}^{\prime}\right\|_{L_{\infty}}<\frac{1-\sin (\mathcal{A}, \mathcal{S})}{1+\sin (\mathcal{A}, \mathcal{S})}
$$

then $M_{n}^{\prime}(\mathcal{A}) \oplus \mathcal{S}^{\perp}=L_{2}$.

Before stating the proof, note that (42) also implies $\left\|1-\mathbf{m}_{n}^{\prime}\right\|_{L_{\infty}}<1$, which by Proposition 1 ensures $M_{n}^{\prime}(\mathcal{A}) \oplus \mathcal{A}^{\perp}=L_{2}$. We also note that since the direct sum condition $\mathcal{A} \oplus \mathcal{S}^{\perp}=L_{2}$ guarantees that $\sin (\mathcal{A}, \mathcal{S})<1$, the norm bound is meaningful as the right-hand side of (42) is positive. In the special case $\mathcal{A} \subseteq \mathcal{S}$ we have $\sin (\mathcal{A}, \mathcal{S})=0$ and (42) becomes $\left\|1-\mathbf{m}_{n}^{\prime}\right\|_{L_{\infty}}<1$ which is the less restrictive requirement of Proposition 1.

Proof: See Appendix III.

We have seen that the initial direct sum condition $\mathcal{A} \oplus \mathcal{S}^{\perp}=$ $L_{2}$ and the curvature bound (42) are sufficient to ensure that the direct sum condition $M_{n}^{\prime}(\mathcal{A}) \oplus \mathcal{S}^{\perp}$ is satisfied. Consequently, by Theorem 2, there is also a unique solution to our nonlinear sampling problem.

As a final remark, note that by relating the curvature bounds (4) with (42) yields

$$
\mathbf{m}^{\prime}(t)>L_{g}=\frac{2 \sin (\mathcal{A}, \mathcal{S})}{1+\sin (\mathcal{A}, \mathcal{S})} \geq 0
$$

and

$$
\mathbf{m}^{\prime}(t)<U_{g}=\frac{2}{1+\sin (\mathcal{A}, \mathcal{S})} \leq 2 .
$$

This means that a sufficient condition for our theory to hold, is to have an ascending nonlinear distortion, with a slope no larger than two. In Section IX, we suggest some extensions of our algorithm to the case in which these conditions are violated.

\section{CONVERGENCE: THE NEWTON APPROACH}

In Sections V and VI, we saw that under the direct sum conditions $M_{n}^{\prime}(\mathcal{A}) \oplus \mathcal{S}^{\perp}=L_{2}$ the iterative oblique projections and the approximated POCS method take the form (23). We now establish that with a small modification, algorithm (23) is guaranteed to converge. Furthermore, it will converge to the input signal $\mathbf{y} \in \mathcal{A}$. 
To this end, we interpret the sequence version of our algorithm (24), as a quasi-Newton method [18], [19], aimed to minimize the consistency cost function

$$
f(a)=\frac{1}{2}\|e(a)\|_{l_{2}}^{2}=\frac{1}{2}\left\|S^{*} M(A a)-c\right\|_{l_{2}}^{2}
$$

where

$$
e(a)=S^{*} M(A a)-c
$$

is the error in the samples with a given choice of $A a \in \mathcal{A}$. Note that by expressing the function $\mathbf{y} \in \mathcal{A}$ in terms of its representation coefficients, we obtained an unconstrained optimization problem.

The true input $\mathrm{y}$ has representation coefficients $a$, for which $f$ attains the global minimum of zero. Since by Theorem 2 there is only one such function, $f$ of (45) has a unique global minimum. Unfortunately, since $M$ is nonlinear, $f$ is in general nonlinear and nonconvex. Obviously, without some knowledge about the global structure of the merit function $f$ (e.g., convexity), optimization methods cannot guarantee to trap the global minimum of $f$. They can, however, find a stationary point of $f$, i.e., a vector $a_{*}$ where the gradient $\nabla f\left(a_{*}\right)$ is zero.

We now establish a key result, showing that if the direct sum conditions are satisfied, then a stationary point of $f$ must also be the global minimum.

Theorem 6: Assume $M_{\mathbf{h}}^{\prime}(\mathcal{A}) \oplus \mathcal{S}^{\perp}=L_{2}$ for all $\mathbf{h} \in L_{2}$. Then a stationary point of $f$ is also its global minimum.

Proof: Assume that $\mathbf{y}_{n}=A a_{n}$ is a stationary point of $f$. Then, the gradient of $f$ at $\mathbf{y}_{n}=A a_{n}$ is $\nabla f\left(a_{n}\right)=\left(S^{*} M_{n}^{\prime} A\right)^{*} e\left(a_{n}\right)=0$. Denoting $\mathbf{x}_{n}=$ $M\left(\mathbf{y}_{n}\right)$ and using definition (46) of $e$, we can also rewrite $\nabla f\left(a_{n}\right)=\left(M_{n}^{\prime} A\right)^{*} S S^{*}\left(\mathbf{x}_{n}-\mathbf{x}_{s}\right)$. Assume to the contrary that $e\left(a_{n}\right)=S^{*}\left(\mathbf{x}_{n}-\mathbf{x}_{s}\right)$ is not the zero vector. Then, since $S^{*}\left(\mathbf{x}_{n}-\mathbf{x}_{s}\right) \in \mathcal{R}\left(S^{*}\right)=\mathcal{N}^{\perp}(S)$, also $\mathbf{s}=S S^{*}\left(\mathbf{x}_{n}-\mathbf{x}_{s}\right)$ is not the zero function within $\mathcal{S}$. By the direct sum $M_{n}^{\prime}(\mathcal{A}) \oplus \mathcal{S}^{\perp}=L_{2}$, we must have that $\nabla f\left(a_{n}\right)=\left(M_{\mathbf{y}_{n}}^{\prime} A\right)^{*} \mathbf{s}$ is not the zero vector, contradicting the fact that a stationary point has been reached.

Note that the combination of Theorems 2 and 6 implies that when the direct sum conditions $M_{\mathbf{h}}^{\prime}(\mathcal{A}) \oplus \mathcal{S}^{\perp}=L_{2}$ are satisfied, optimization methods which are able to trap stationary points of $f$, also retrieve the true input signal $\mathbf{y} \in \mathcal{A}$. Also, in Theorem 5 we have obtained simple sufficient conditions for these direct sums to hold. This leads to the following corollary.

Corollary 1: Assume that the slope of the nonlinear distortion satisfies

$$
\left\|1-\mathbf{m}^{\prime}\right\|_{L_{\infty}}<\frac{1-\sin (\mathcal{A}, \mathcal{S})}{1+\sin (\mathcal{A}, \mathcal{S})}
$$

and that $\mathcal{A} \oplus \mathcal{S}^{\perp}=L_{2}$. Then any algorithm which can trap a stationary point $a_{*}$ of $f$ in (45) also recovers the true input $\mathbf{y}=A a_{*}$.
We now interpret (24) as a quasi-Newton method aimed to minimize the cost function $f$ of (45). For descent methods in general, and quasi-Newton specifically, we iterate

$$
a_{n+1}=a_{n}+\alpha_{n} p_{n}
$$

with $\alpha_{n}$ and $p_{n}$ being the step size and search direction, respectively. To minimize $f$, the search direction is set to be a descent direction:

$$
\left\langle p_{n}, \nabla f\left(a_{n}\right)\right\rangle_{l_{2}}<0
$$

where, $p_{n}=-B_{n} \nabla f\left(a_{n}\right)$ for some positive-definite matrix ${ }^{3}$ $B_{n}$. The step size $\alpha_{n}$ is chosen to satisfy the Wolfe conditions [18] (also known as the Armijo and curvature conditions) by using the following simple backtracking procedure:

$$
\begin{aligned}
& \text { Set } \alpha_{n}=1 \\
& \text { Repeat until } f\left(a_{n+1}\right) \leq f\left(a_{n}\right)+\frac{1}{4} \alpha_{n}\left\langle p_{n}, \nabla f\left(a_{n}\right)\right\rangle_{l_{2}} \\
& \text { by setting } \alpha_{n}=\frac{1}{2} \alpha_{n} .
\end{aligned}
$$

It is easy to see that the sequence version of our algorithm (24) is in the form (48) with $\alpha_{n}=1$ and $p_{n}=-\left(S^{*} M_{n}^{\prime} A\right)^{\dagger} e\left(a_{n}\right)$. This search direction is gradient related:

$$
\begin{aligned}
\left\langle\nabla f\left(a_{n}\right), p_{n}\right\rangle_{l_{2}} & =-\left\langle e\left(a_{n}\right), S^{*} M_{n}^{\prime} A\left(S^{*} M_{n}^{\prime} A\right)^{\dagger} e\left(a_{n}\right)\right\rangle_{l_{2}} \\
& =-\left\|P_{\mathcal{R}\left(S^{*} M_{n}^{\prime} A\right)} e\left(a_{n}\right)\right\|_{l_{2}}^{2} \\
& =-\left\|e\left(a_{n}\right)\right\|_{l_{2}}^{2} .
\end{aligned}
$$

The last equality follows from the fact that when $M_{n}^{\prime}(A) \oplus \mathcal{S}^{\perp}=$ $L_{2}$ is satisfied then [14] $\mathcal{R}\left(S^{*} M_{n}^{\prime} A\right)=\mathcal{R}\left(S^{*}\right)$, and by (46), $e\left(a_{n}\right) \in \mathcal{R}\left(S^{*}\right)$.

Using this Newton point of view, we now suggest a slightly modified version of our algorithm, which converges to coefficients $a_{*}$ of the true input $\mathbf{y}=A a_{*}$.

Theorem 7: The algorithm of Table I will converge to coefficients $a_{*}$ of the true input, if

1) $\mathcal{A} \oplus \mathcal{S}^{\perp}=L_{2}$ and the derivative $\mathbf{m}^{\prime}(t)$ satisfies the bound $\left\|1-\mathbf{m}^{\prime}\right\|_{L_{\infty}}<(1-\sin (\mathcal{A}, \mathcal{S})) /(1+\sin (\mathcal{A}, \mathcal{S}))$;

2) $\mathbf{m}^{\prime}(t)$ is Lipschitz continuous.

Before stating the proof, note that condition 1 implies $M_{n}^{\prime}(\mathcal{A}) \oplus \mathcal{S}^{\perp}=L_{2}$ for all $\mathbf{y}_{n}$. The only difference with the basic version (24) of the algorithm, is by introducing a step size $\alpha_{n}$ and stating requirement 2 . The latter technical condition is needed to assure convergence of descent based methods.

Proof: By Corollary 1 we only need to show that the algorithm converges to a stationary point of $f$. We start by following known techniques for analyzing Newton-based iterations. The

${ }^{3}$ With quasi-Newton methods $p_{n}=-B_{n} \nabla f\left(a_{n}\right)$ where $B_{n}$ is a computationally efficient approximation of the Hessian inverse. Though in our setup it is possible to show that $p_{n}$ is related to $\nabla f\left(a_{n}\right)$ with a matrix approximating the Hessian inverse, we will not claim for computational efficiency here. Nonetheless, we will use the term quasi-Newton when describing our method. 
TABLE I

THE PRoposed ALGORITHM

Initialize $a_{0}=0$

- Iterate

$$
a_{n+1}=a_{n}-\alpha_{n}\left(S^{*} M_{n}^{\prime} A\right)^{\dagger}\left(S^{*} M\left(A a_{n}\right)-c\right)
$$

with a step size $\alpha_{n}$ chosen by (50).

- Denoting the convergent sequence by $a_{*}$, reconstruct the signal as $\mathbf{y}=A a_{*}$.

standard analysis is to show that Zoutendijk condition [18] is satisfied:

$$
\sum_{n=0}^{\infty} \cos ^{2} \theta_{n}\left\|\nabla f\left(a_{n}\right)\right\|_{l_{2}}^{2}<\infty
$$

where

$$
\cos \theta_{n}=-\frac{\left\langle\nabla f\left(a_{n}\right), p_{n}\right\rangle_{l_{2}}}{\left\|\nabla f\left(a_{n}\right)\right\|_{l_{2}}\left\|p_{n}\right\|_{l_{2}}}
$$

is the cosine of the angle between the gradient $\nabla f\left(a_{n}\right)$ and the search direction $p_{n}$. If

$$
\cos \theta_{n} \geq \epsilon>0
$$

that is, the search direction never becomes perpendicular to the gradient, then Zoutendijk condition implies that $\nabla f\left(a_{n}\right) \rightarrow 0$, so that a stationary point is reached.

To guarantee (52) we rely on the following lemma.

Lemma 3 [18, Theorem 3.2]: Consider iterations of the form (48), where $p_{n}$ is a descent direction and $\alpha_{n}$ satisfies the Wolfe conditions. If $f$ is bounded below, continuously differentiable and $\nabla f$ is Lipschitz continuous then (52) holds.

In our problem, $p_{n}$ is a descent direction and the backtracking procedure guarantees that the step size $\alpha_{n}$ satisfies the Wolfe conditions [18], [19]. Also, $f(a)=1 / 2\|e(a)\|_{l_{2}}^{2} \geq 0$ and the partial derivatives of $f$ with respect to $a$ exist and are given by $\nabla f(a)=\left(S^{*} M_{n}^{\prime} A\right)^{*} e(a)$. Thus, all that is left is to prove Lipschitz continuity of $\nabla f$ (which will also imply that $\nabla f$ is a continuous mapping, and thus, $f$ is continuously differentiable). This is proven in Appendix IV.

We now establish (54). Using (51)

$$
\cos \theta_{n}=\frac{\left\|e_{n}\right\|_{l_{2}}^{2}}{\left\|Q_{n}^{*} e_{n}\right\|_{l_{2}}\left\|Q_{n}^{\dagger} e_{n}\right\|_{l_{2}}} \geq \frac{1}{\left\|Q_{n}\right\|\left\|Q_{n}^{\dagger}\right\|}
$$

where we used the notations $Q_{n}=S^{*} M_{n}^{\prime} A, e_{n}=e\left(a_{n}\right)$. Since $\left\|Q_{n}\right\| \leq U_{S} U_{g} U_{A}$, it is sufficient to show that $\left\|Q_{n}^{\dagger}\right\|$ is upper bounded. Now [29], $\left\|Q_{n}^{\dagger}\right\|=\left(\gamma\left(Q_{n}\right)\right)^{-1}$, where

$$
\gamma\left(Q_{n}\right)=\inf _{b \in \mathcal{N}^{\perp}\left(Q_{n}\right),\|b\|_{l_{2}}=1}\left\|Q_{n} b\right\|_{l_{2}} .
$$

Since $M_{n}^{\prime}(\mathcal{A}) \oplus \mathcal{S}^{\perp}=L_{2}$ for all $\mathbf{y}_{n}$, there exists an $\tilde{\epsilon}>0$ such that $\cos \left(M_{n}^{\prime}(\mathcal{A}), \mathcal{S}\right) \geq \tilde{\epsilon}$ for all $n$. Therefore,

$$
\begin{aligned}
\left\|Q_{n} b\right\|_{l_{2}} & =\left\|S^{*} P_{\mathcal{S}} M_{n}^{\prime} A b\right\|_{l_{2}} \\
& \geq L_{S} \cos \left(M_{n}^{\prime}(\mathcal{A}), \mathcal{S}\right)\left\|M_{n}^{\prime} A b\right\|_{L_{2}} \\
& \geq L_{S} \tilde{\epsilon} L_{g} L_{A}\|b\|_{l_{2}}
\end{aligned}
$$

so that $\gamma\left(Q_{n}\right) \geq L_{S} \tilde{\epsilon} L_{g} L_{A}$ and (54) is satisfied. Having proved that the suggested quasi-Newton algorithm converges to a stationary point of $f$, by Theorem 6 , we also perfectly reconstruct the coefficients of the input $\mathbf{y}$.

\section{PRACTICAL CONSIDERATIONS}

We have presented a Newton based method for perfect reconstruction of the input signal. Though the suggested algorithm has an interesting interpretation in terms of iterative oblique projections and approximated POCS, it is definitely not the only choice of an algorithm one can use. In fact, any optimization method, which is known to trap a stationary point of the consistency merit function (45), will, by Theorem 6 , also trap its (unique) global minimum of zero. Thus, we presented here conditions on the sampling space $\mathcal{S}$, the restoration space $\mathcal{A}$ and the nonlinear distortion $M$, for which minimization of the merit function (45) leads to perfect reconstruction of the input signal.

We will now explain how some of the conditions on the spaces involved and the nonlinear distortion can be relaxed.

For some applications, the bounds (43) and (44) might not be satisfied everywhere but only for a region of input amplitudes. For example, the mapping $M(\mathbf{y})(t)=\mathbf{y}^{3}(t)$ is Lipschitz continuous only on a finite interval. Also, the derivative $\mathbf{m}^{\prime}$ is zero for an input amplitude of zero. Thus, conditions (43) and (44) are violated unless we restrict our attention to input functions $\mathbf{y} \in \mathcal{A}$ which are a priori known to have amplitudes within some predefined, sufficiently small interval. Restricting our attention to amplitude bounded signals can be obtained by minimizing (45) with constraints of the form $\alpha_{1} \leq \mathbf{y}(t) \leq \alpha_{2}$. There are many methods of performing numerical optimization with constraints. For example, one common approach is to use Newton iterations while projecting the solution at each step onto the feasible set (e.g., [30]). Another example is the use of barrier methods [18].

Note, however, that the functional (45) is optimized with respect to the representation coefficients $a$ and not the continuous-time signal $\mathbf{y}$ itself. Thus, it is imperative to link amplitude bounds on $\mathbf{y}$ to its representation coefficients $a$, in cases where amplitude constraints should be incorporated. It is possible to do that if we also assume that the reconstruction space $\mathcal{A}$ is a reproducing kernel Hilbert space (RKHS) [31], [32], which are quite common in practice. For example, any shift-invariant frame of $L_{2}$ corresponds to a RKHS [33]. In particular, the subspace of band-limited functions is a RKHS. Formally, if $\mathcal{A}$ is a RKHS, then for any $\mathbf{y} \in \mathcal{A} \subseteq L_{2},\left|\mathbf{y}\left(t_{0}\right)\right|=\left|\left\langle\mathbf{k}_{t_{0}}, \mathbf{y}\right\rangle\right| \leq$ $\left\|\mathbf{k}_{t_{0}}\right\|_{L_{2}}\|\mathbf{y}\|_{L_{2}}$, where $\mathbf{k}$ is the kernel function of the space [31], [32]. Thus, we can bound the amplitude of $\mathbf{y}$ by controlling its energy, which can be accomplished by controlling the norm of its representation coefficients. 


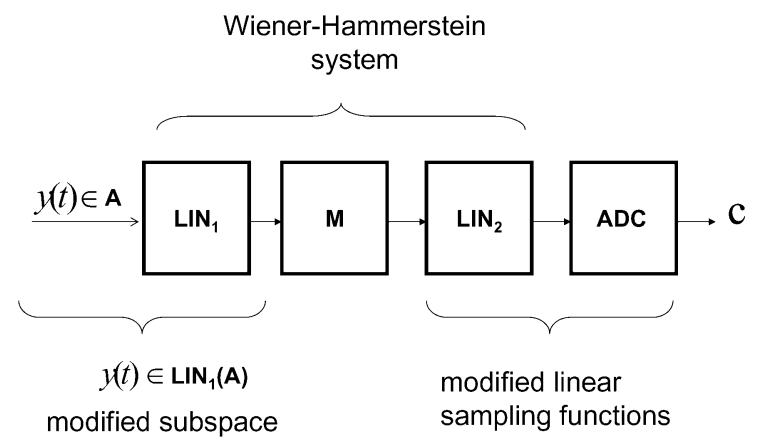

Fig. 6. Extension of the memoryless nonlinear setup to Wiener-Hammerstein sampling systems.

An additional observation concerns the special case of $\mathcal{S}=$ $\mathcal{A}$. In such a setup, at the $n$th approximation point, the gradient of (45) is $\nabla f\left(a_{n}\right)=\left(A^{*} M_{n}^{\prime} A\right)^{*} e\left(a_{n}\right)$. Since the operator $A^{*} M_{n}^{\prime} A$ is positive, we can use $-e\left(a_{n}\right)$ as a descent direction, eliminating the need to compute oblique projections at each step of the algorithm.

We have also assumed that the nonlinearity is defined by a strictly ascending functional $\mathbf{m}(t)$. If $\mathbf{m}$ is strictly descending then we can always invert the sign of our samples, i.e., process the sequence $-c$ instead of $c$, to mimic the case where we sample $-\mathbf{x}(t)=-\mathbf{m}(\mathbf{y}(t))$, instead of $\mathbf{x}$. Once convergence of the algorithm is obtained, the sign of the resulting representation coefficients should be altered. Thus, our algorithm and the theory behind it, also apply to strictly descending nonlinearities.

Finally, we point out that the developed theory imposed the nonlinearity $\mathbf{m}$ to have a slope $\mathbf{m}^{\prime}$ which is no larger than the upper bound $U_{g} \leq 2$. This is, however, merely a sufficient condition. In practice, we have also simulated nonlinearities with a larger slope, and the algorithm still converged (see the examples within Section $\mathrm{X}$ ).

\section{A. Extension to Wiener-Hammerstein Systems}

Throughout the paper we have assumed that the nonlinear distortion caused by the sensor is memoryless. Such a model is a special case of Wiener, Hammerstein and Wiener-Hammerstein systems [21]. A Wiener system is a composition of a linear mapping followed by a memoryless nonlinear distortion, while in a Hammerstein model these blocks are connected in reverse order. Wiener-Hammerstein systems combine the above two models, by trapping the static nonlinearity, with dynamic and linear models from each side. We can address such systems by noting that we can absorb the first linear mapping into the structural constraints $\mathbf{y} \in \mathcal{A}$, and use the last linear operator to define a modified set of generalized sampling functions. Thus, it is possible to extend our derivations to Wiener-Hammerstein acquisition devices as well. This concept is illustrated in Fig. 6.

\section{Simulations}

In this section, we simulate different setups of reconstructing a signal in a subspace, from samples obtained by a nonlinear and nonideal acquisition process.

\section{A. Band-limited Example}

We start by simulating an optical sampling system described in [34]. There, the authors implement an acquisition device

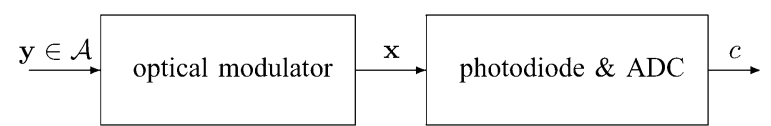

Fig. 7. Optical sampling system. For high-gain signals, the optical modulator introduces nonlinear amplitude distortions.

receiving a high frequency, narrowband electrical signal. The signal is then converted to its baseband using an optical modulator. In [34] a small-gain electrical signal is used, such that the transfer function of the optical modulator is approximately linear. Here, however, we are interested in the nonlinear distortion effects. Thus, we simulate an example of a high-gain input signal, such that the optical modulator exhibits memoryless nonlinear distortion effects when transforming the electrical input $\mathbf{y}$ to an optical output. The sampling system is shown in Fig. 7 .

We note that the ability to introduce high-gain signals is very important in practice as it allows to increase the dynamic range of a system, improving the signal to interference ratio. We now show that by applying the algorithm of Theorem 7, we are able to eliminate the nonlinear effects caused by the optical modulator.

The input-output distortion curve of the optical modulator is known in advance, and can be described by a sine wave [35]. Here, we simulate a nonlinear distortion which is given by $\mathbf{m}(t)=\sin (5 t)$. For input signals $\mathbf{y}$ which satisfy $\|\mathbf{y}\|_{L_{\infty}}<\pi / 10$ the optical modulator introduces a strictly ascending distortion curve. Notice, however, that to test a practical scenario, we apply our method to a nonlinear distortion having a maximal slope of five, which is larger than the bound (44). In this simulation the input signal $\mathbf{y}$ is composed of two high frequency, narrowband components; a contribution at a carrier frequency of $f_{0}=550 \mathrm{MHz}$ and at $f_{1}=600 \mathrm{MHz}$. The bandwidth of each component is set to $8 \mathrm{MHz}$. Hence, the input $\mathbf{y}$ lies in a subspace $\mathcal{A}$ spanned by $\left\{\operatorname{sinc}(t / T-n) \cos \left(2 \pi f_{0} t\right)\right\}_{n}$ and $\left\{\operatorname{sinc}(t / T-n) \cos \left(2 \pi f_{1} t\right)\right\}_{n}$, where $T^{-1}=16 \mathrm{MHz}$. The support of the input signal (in the Fourier domain) is depicted in Fig. 8(a).

As the input signal is acquired by the nonlinear optical sensor, the input-output sine distortion curve introduces odd order harmonics at the output. Already the third order harmonics contribute energy in the region of $1.5-2 \mathrm{GHz}$. The resulting signal is sampled by an (approximately linear) optical detector (photodiode) and an electrical ADC. We approximate both these stages by an antialiasing low-pass filter followed by an ideal sampler, similar to the description of Fig. 2. The antialiasing filter is chosen to have a transition band in the range $620 \mathrm{MHz}-1 \mathrm{GHz}$, and the sampling rate is $2 \mathrm{GHz}$. Thus, in compliance with Fig. 2, the sampling functions $\mathbf{s}\left(t-n T_{2}\right)$ are shifted and mirrored versions of the impulse response of this antialiasing filter, with $T_{2}^{-1}=2 \mathrm{GHz}$. The original input, the resulting output $\mathbf{x}=$ $M(\mathbf{y})$ and the frequency response of the antialiasing filter are shown in Fig. 8(b). Notice the harmonics in $\mathbf{x}$, introduced by the optical modulator. Also note that the sampling rate of $2 \mathrm{GHz}$, is below the Nyquist rate of $\mathbf{x}$.

Since we have prior knowledge of $\mathbf{y}$ being an element of $\mathcal{A}$ (here, a subspace composed of two narrowband regions around 


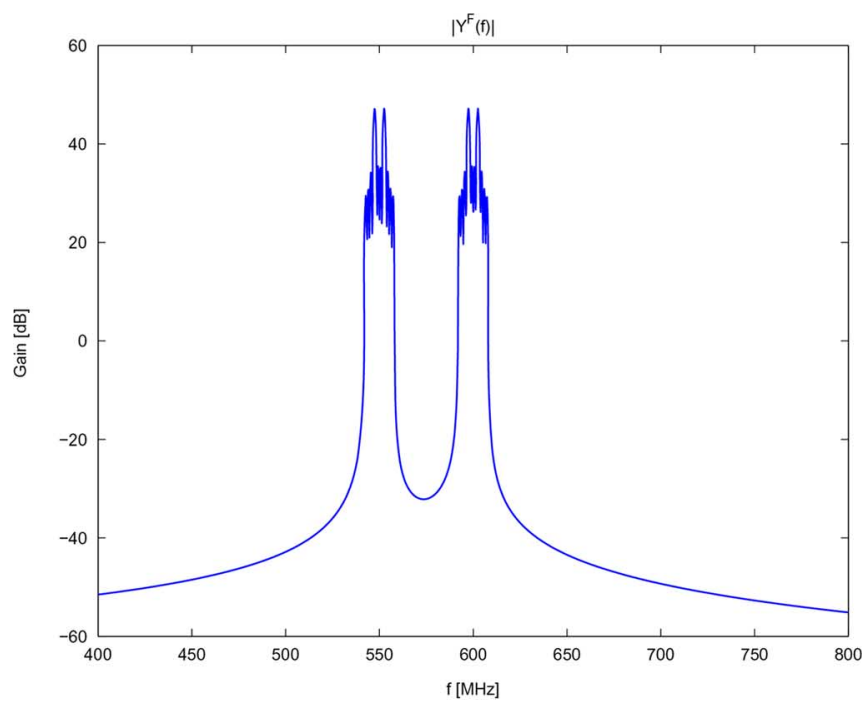

(a)

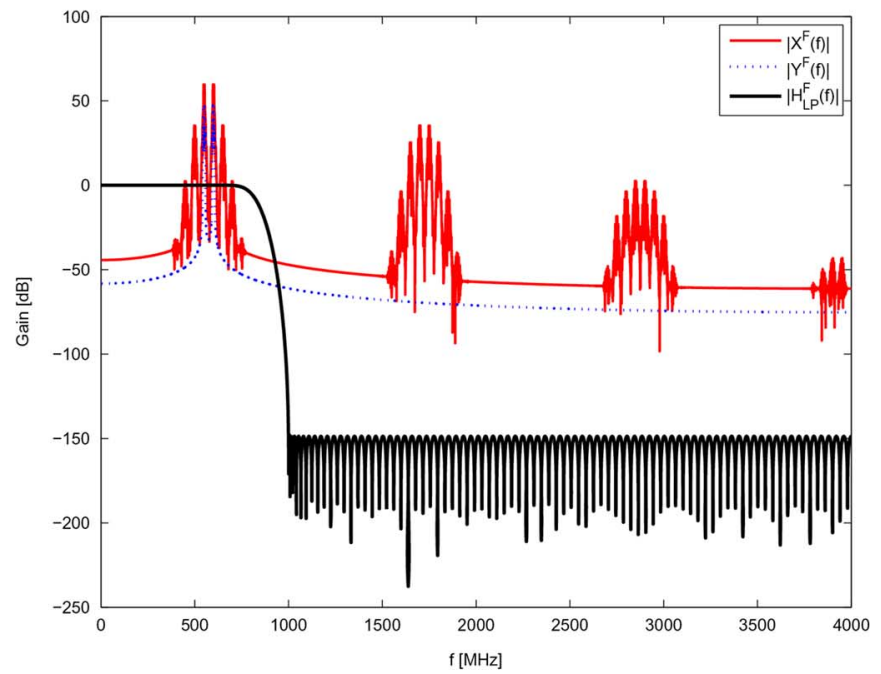

(b)

Fig. 8. (a) Input signal $\mathbf{y}$ is composed of two high-frequency components, modulated at 550 and $600 \mathrm{MHz}$. (b) The input signal $\mathbf{y}$, the distorted output $\mathbf{x}=M(\mathbf{y})$, and the frequency response of the antialiasing filter serving as the generalized sampling function.

550 and $600 \mathrm{MHz}$ ) we are able to apply the algorithm of Theorem 7. In Fig. 9(a), we show the true input $\mathbf{y}(t)$ and its approximation $\mathbf{y}_{1}(t)$ obtained by a single iteration of the algorithm. In Fig. 9(b) we show the result after the third iteration. The consistency error in the samples appears in the title of each figure. At the seventh iteration the algorithm has converged (to the true input signal), within the numerical precision of the machine.

If we disregard the nonlinearity (i.e., by assuming that $M=$ $I)$, then the solution will be to perform an oblique projection onto the reconstruction space: $\hat{\mathbf{y}}=P_{\mathcal{A}, \mathcal{S}^{\perp}} \mathbf{x}_{s}$. Since we initialize our algorithm with $\mathbf{y}_{0}=0$, it is simple to show that $\mathbf{y}_{1}=\hat{\mathbf{y}}=P_{\mathcal{A}, \mathcal{S}^{\perp}} \mathbf{X}_{s}$, as shown in Fig. 9(a). Evidently, accounting for the nonlinearity improves this result.

Another possibility is to assume that the samples are ideal, i.e., to assume that $c$ are pointwise evaluations of the function $\mathbf{x}$, and apply $M^{-1}(c)$ prior to interpolation. Unfortunately, since in practice the samples are nonideal, many of them receive values

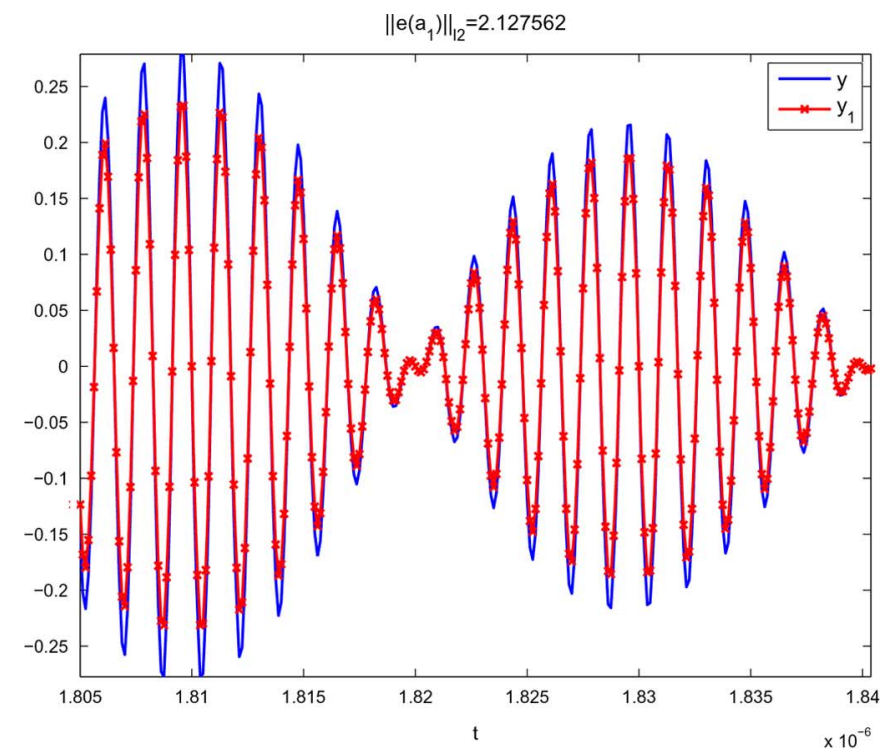

(a)

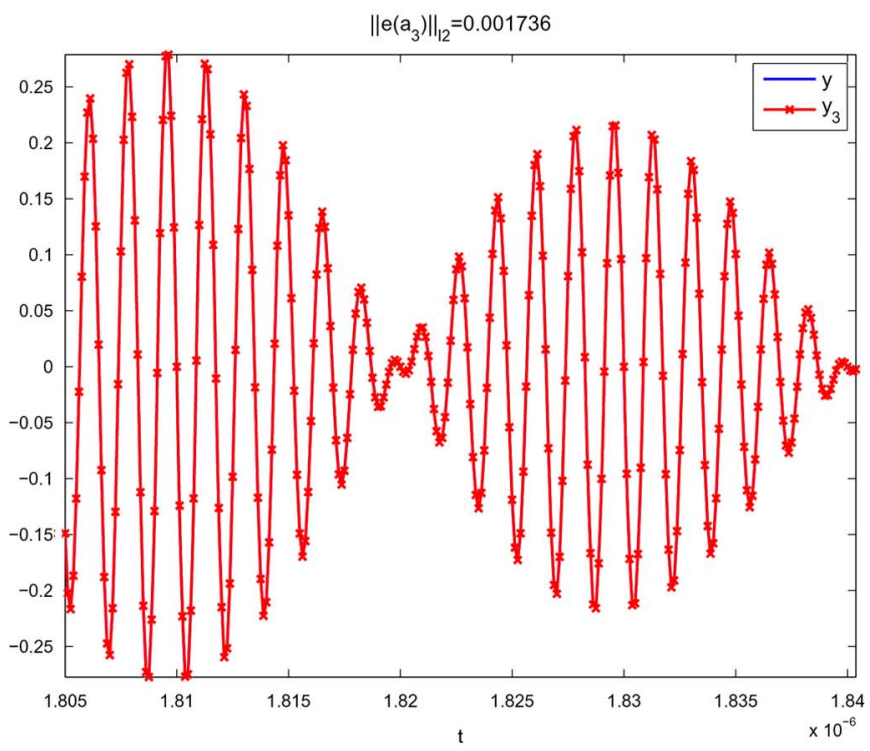

(b)

Fig. 9. (a) True input signal $\mathbf{y}$, and its approximation $\mathbf{y}_{1}$ obtained by a single iteration of the algorithm. (b) True input signal $\mathbf{y}$, and its approximation $\mathbf{y}_{3}$ obtained at the third iteration. The consistency error is stated in the title of each plot.

outside the range $[-1,1]$. In that case, the operation $M^{-1}(c)$ cannot even be performed, since the domain of the arcsin function is restricted to the $[-1,1]$ interval. One can then use an add-hoc approach by normalizing the samples which have excessive values to $c[n] /|c[n]|$. As evident from the time and frequency-domain plots of Fig. 10, this approach results in poor approximation of the input signal, giving in this example a spurious-free dynamic rage of only $16 \mathrm{~dB}$. Our proposed method, in contrast, perfectly reconstructs the input signal, theoretically giving an infinite spurious-free dynamic range.

\section{B. Non-Band-Limited Example}

As another example, which departs from the band-limited setup, assume that the input signal $\mathbf{y}$ lies in a shift-invariant 


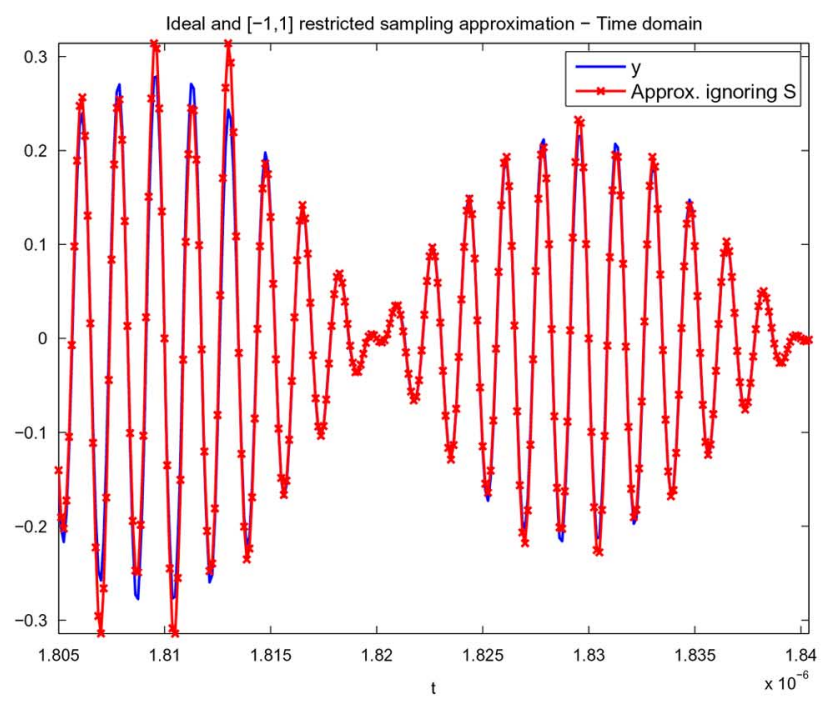

(a)

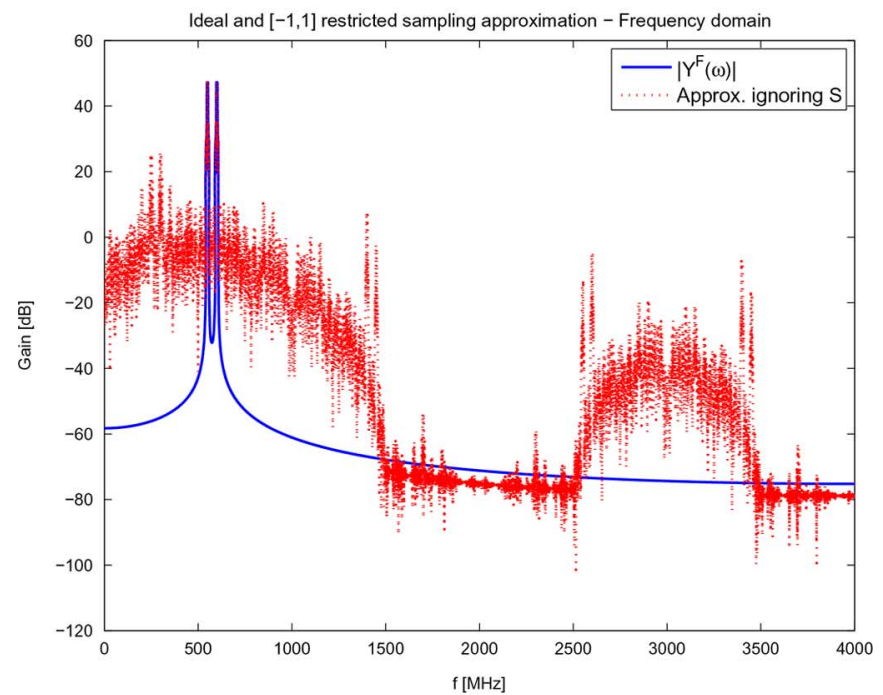

(b)

Fig. 10. True input signal $\mathbf{y}$, and its approximation, obtained by (falsely) assuming ideal samples, restricted to the $[-1,1]$ interval. (a) Time-domain plot. (b) Frequency-domain plot.

subspace, spanned by integer shifts of the generator $\mathbf{a}(t)=$ $e^{-t} u(t)$, where $u(t)$ is the unit step function. For example, $\mathbf{a}(t)$ can be the impulse response of an RC circuit, with the delay constant RC set to one. We choose the nonlinear distortion as the inverse tangent function, i.e., $\mathbf{x}(t)=\operatorname{atan}(\mathbf{y}(t))$, and the sampling scheme is given by local averages of the form $c[n]=$ $\int_{n-1}^{n} \mathbf{x}(t) d t$. Accordingly, the sampling space is also shift invariant, with the generator $\mathbf{s}(t)=u(t)-u(t-1)$. In Fig. 11(a) we show the original input signal $\mathbf{y}(t)$ and two naive forms for approximating it from the nonlinear and nonideal samples. First, one can neglect the nonlinear effects of the acquisition device, assuming that $M$ is the identity mapping. As with the previous example, in this case the approximation takes the form of an oblique projection onto the reconstruction space along $\mathcal{S}^{\perp}$, which also results from the first iteration of our algorithm. As another option, one can assume an ideal sampling scheme. In that case, $M^{-1}(c)$ are assumed to be the ideal samples of $\mathbf{y}$, from which the signal is reconstructed. As can be seen from

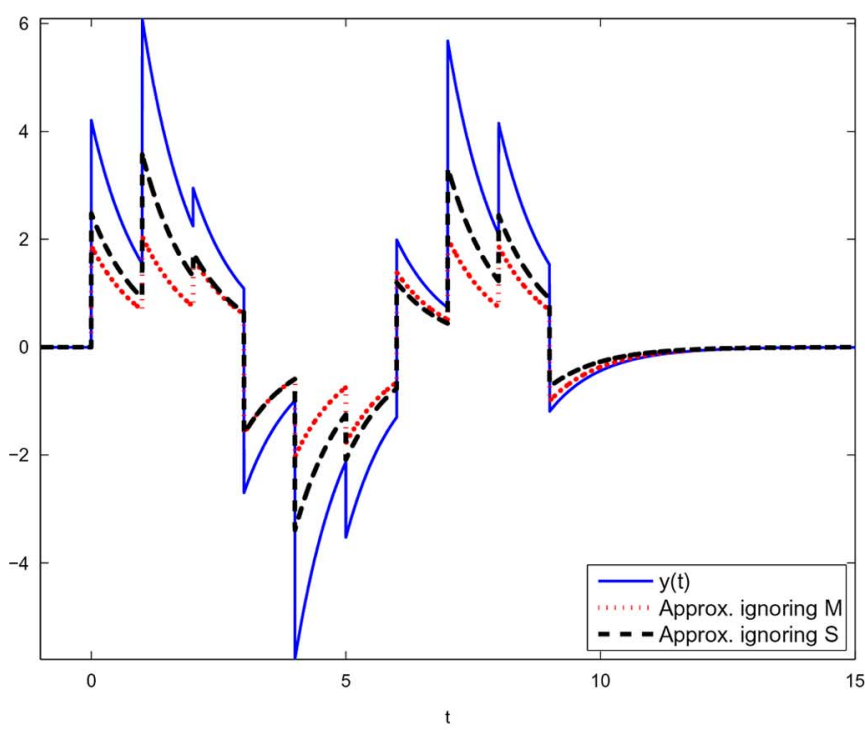

(a)

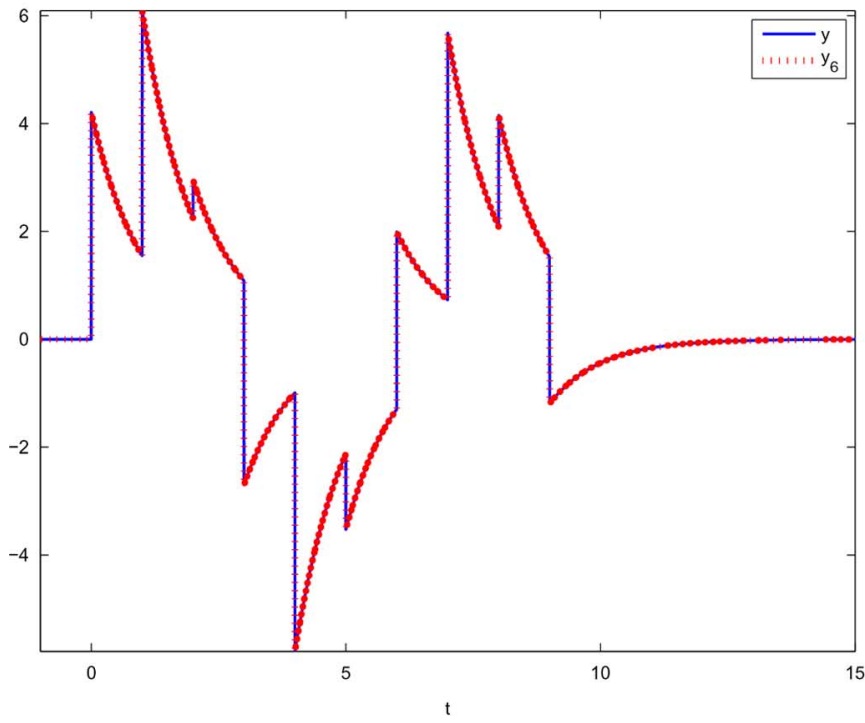

(b)

Fig. 11. (a) Original input $\mathbf{y}(t)$ (solid), and two forms of approximation: Ignoring the nonlinearity (dotted) and ignoring the nonideality of the sampling scheme (dashed). (b) True input signal $\mathbf{y}$, and its approximation $\mathbf{y}_{6}$ obtained at the sixth iteration.

the figure, this also leads to inexact restoration of the input. On the other hand, our algorithm takes into account the nonlinear distortion and the nonideality of the sampling scheme, yielding perfect reconstruction of the signal [Fig. 11(b)].

Though out of the scope of the developed theory, it is interesting to investigate the influence of quantization noise. For this purpose, we repeat the last simulation, when quantizing the samples with a quantization step of 0.1 . Naturally, in such a setup there is no reason to expect perfect reconstruction of the input signal. Instead, we will be satisfied if an approximation $\hat{\mathbf{y}} \in \mathcal{A}$ is found, which can explain the nonlinear, nonideal and quantized sample sequence. For that purpose, our algorithm was slightly rectified by quantizing at each iteration the samples error vector $S^{*} M\left(A a_{n}\right)-c$ according to the quantization step. The results of this simulation are presented in Fig. 12. As can be seen from the 


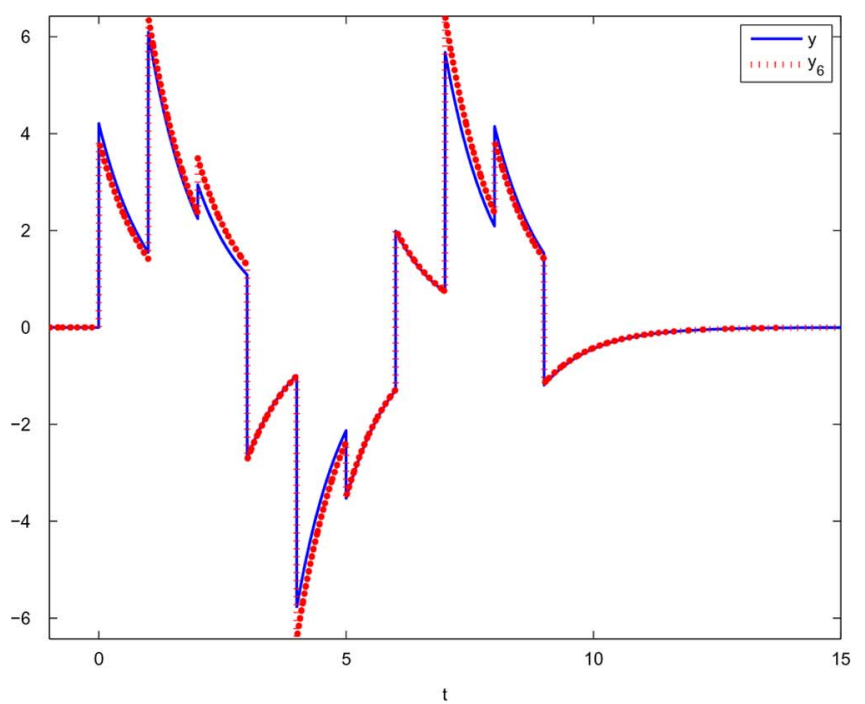

(a)

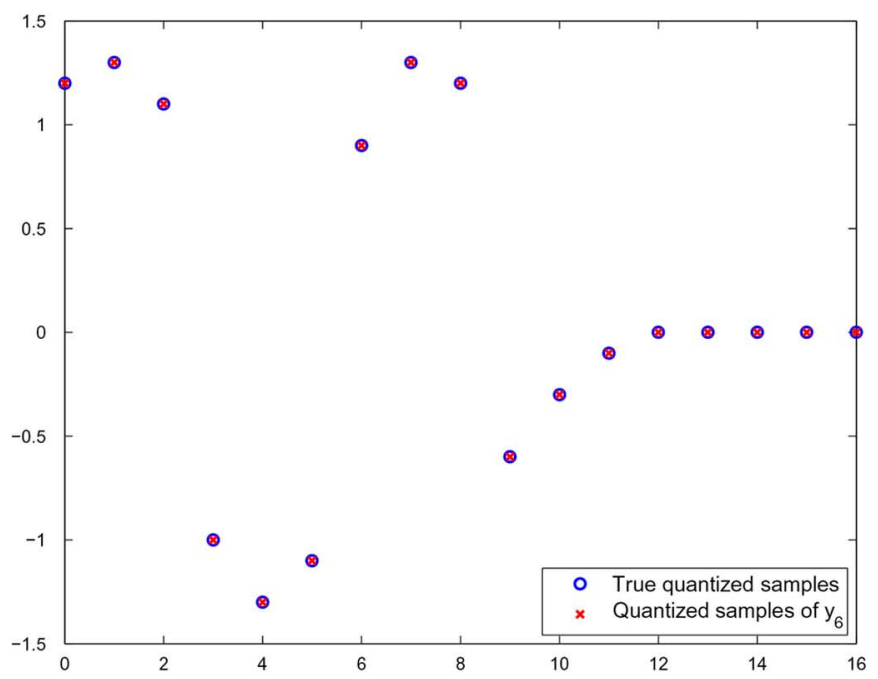

(b)

Fig. 12. Nonlinear, generalized and quantized sampling case. (a) True input signal $\mathbf{y}$, and its approximation $\mathbf{y}_{6}$ obtained at the sixth iteration (dashed). (b) True quantized samples, and the quantized sample sequence of the approximation $\mathbf{y}_{6}$.

figure, the approximation is not identical to the input function $\mathbf{y}$, yet, they both produce the same quantized sample sequence. We emphasize, however, that the developed theory does not take noisy samples into account, and we do not claim that this modified algorithm will produce consistent approximations under all circumstances.

\section{CONCLuSION}

In this paper, we addressed the problem of reconstructing a signal in a subspace from its generalized samples, which are obtained after the signal is distorted by a memoryless nonlinear mapping. Assuming that the nonlinearity is known, we derived sufficient conditions on the nonlinear distortion curve and the spaces involved, which ensure perfect reconstruction of the input signal from these nonlinear and nonideal samples. The developed theory shows that for such setups, the problem of perfect reconstruction can be resolved by retrieving stationary points of a consistency cost function. We then developed an algorithm for this problem, which was also demonstrated by simulations. Finally, we explained how to extend these derivations to nonlinear and nonideal acquisition devices which can be modeled by a Wiener-Hammerstein system. Future extensions of this research may consider the important problem of estimating the nonlinearity of the system from the known samples, and approximating the signal from noisy samples.

\section{APPENDIX I \\ PROOF OF THEOREM 1}

Under the conditions of the theorem, $\mathbf{x}=M(\mathbf{y})$ admits a Fourier series expansion, and can be written as $\mathbf{x}=\sum_{m} \beta[m] e^{j \omega_{0} m t}$ for some Fourier coefficients $\beta$. By (6), we then have

$$
\begin{aligned}
c[n] & =\int \sum_{m} \beta[m] e^{j \omega_{0} m t} \mathbf{s}(t) e^{-j \omega_{0} n t} d t \\
& =\sum_{m} \beta[m] \mathbf{S}^{F}\left(\omega_{0}(n-m)\right) .
\end{aligned}
$$

Thus, the Fourier coefficients $\beta$ of $\mathbf{x}$ are related to the generalized samples $c$ through discrete-time convolution $c=\beta * \mathbf{h}$ with $h[n]=\mathbf{S}^{F}\left(\omega_{0} n\right)$.

To stably reconstruct $\beta$ from the sample sequence $c$ we need the discrete-time Fourier transform (DTFT) of the sequence $h$ to be invertible and bounded. Since the continuous-time Fourier transform of $\mathbf{S}^{F}(\omega)$ at $\mu$ is $\int_{-\infty}^{\infty} S(\omega) e^{-j \omega \mu} d \omega=2 \pi s(-\mu)$, then $h$, being the uniform samples of $\mathbf{S}^{F}(\omega)$ with interval $\omega_{0}=$ $2 \pi / T_{0}$, has the DTFT

$$
\frac{1}{\omega_{0}} \sum_{k} 2 \pi \mathbf{s}\left(\frac{-(\mu+2 \pi k)}{\omega_{0}}\right) ; \quad \forall \mu \in[0,2 \pi] .
$$

Replacing $t=-\mu / \omega_{0}$, we obtain that (57) is bounded from below and above if and only if (12) holds.

The coefficients $\beta$ can now be determined, by convolving $c$ with $h^{-1}$. Finally, the restoration of $\mathbf{y}$ takes the form (13).

\section{APPENDIX II PROOF OF THEOREM 4}

Before stating the proof, we need the following lemma, which shows that the direct sum condition is invariant under linear continuous and invertible mappings. Throughout this section, we use the more general notation of inner products and norms within some Hilbert space $\mathcal{H}$ (not necessarily $\mathcal{H}=L_{2}$ ).

Lemma 4: Let $T$ be a linear, continuous and continuously invertible mapping. Then $\mathcal{A} \oplus \mathcal{S}^{\perp}=\mathcal{H}$ if and only if $T(\mathcal{A}) \oplus$ $T\left(\mathcal{S}^{\perp}\right)=\mathcal{H}$.

Proof: Assume $\mathcal{A} \oplus \mathcal{S}^{\perp}=\mathcal{H}$. If there is some $0 \neq \mathbf{f} \in$ $T(\mathcal{A}) \cap T\left(\mathcal{S}^{\perp}\right)$, then $0 \neq T^{-1} \mathbf{f} \in \mathcal{A} \cap \mathcal{S}^{\perp}$ contradicting the assumption $\mathcal{A} \cap \mathcal{S}^{\perp}=\{0\}$. To show $T(\mathcal{A})+T\left(\mathcal{S}^{\perp}\right)=\mathcal{H}$, take some $\mathbf{f} \in \mathcal{H}$. Then there is a decomposition $T^{-1} \mathbf{f}=\mathbf{f}_{a}+\mathbf{f}_{v}$ for some $\mathbf{f}_{a} \in \mathcal{A}, \mathbf{f}_{v} \in \mathcal{S}^{\perp}$. Thus, $T \mathbf{f}_{a} \in T(\mathcal{A})$ and $T \mathbf{f}_{v} \in T\left(\mathcal{S}^{\perp}\right)$ 
constitute a decomposition of $\mathbf{f}$. The proof in the other direction is similar.

By Lemma $4, \mathcal{S}^{\perp} \oplus M_{n}^{\prime}(\mathcal{A})=\mathcal{H}$ if and only if $\left(M_{n}^{\prime}\right)^{-1}\left(\mathcal{S}^{\perp}\right) \oplus \mathcal{A}=\mathcal{H}$. Furthermore, $\left(M_{n}^{\prime}\right)^{-1}\left(\mathcal{S}^{\perp}\right) \oplus \mathcal{A}=\mathcal{H}$ implies [12], [16]

$\sin \left(\mathcal{A},\left(M_{n}^{\prime-1}\left(\mathcal{S}^{\perp}\right)\right)^{\perp}\right)=\sup _{\mathbf{f} \in \mathcal{A},\|\mathbf{f}\|=1}\left\|P_{\left(M_{n}^{\prime}\right)^{-1}\left(\mathcal{S}^{\perp}\right)} \mathbf{f}\right\|<1$.

Noting that $\left(M_{n}^{\prime}\right)^{-1}\left(\mathcal{S}^{\perp}\right)=\mathcal{N}\left(S^{*} M_{n}^{\prime}\right)$ we conclude that the mapping $P_{\mathcal{N}\left(S^{*} M_{n}^{\prime}\right)} P_{\mathcal{A}}$ is truncating. Thus, for any initialization $\mathbf{h}_{n}^{0}$,

$$
\left(P_{\mathcal{N}\left(S^{*} M_{n}^{\prime}\right)} P_{\mathcal{A}}\right)^{\infty} \mathbf{h}_{n}^{0}=0
$$

and

$$
\sum_{i=0}^{\infty}\left(P_{\mathcal{N}\left(S^{*} M_{n}^{\prime}\right)} P_{\mathcal{A}}\right)^{i}=\left(I-P_{\mathcal{N}\left(S^{*} M_{n}^{\prime}\right)} P_{\mathcal{A}}\right)^{-1}
$$

Combining (58) and (59) with (31) gives

$$
\mathbf{y}_{n+1}=\left(I-P_{\mathcal{N}\left(S^{*} M_{n}^{\prime}\right)} P_{\mathcal{A}}\right)^{-1}\left(S^{*} M_{n}^{\prime}\right)^{\dagger} r_{n}
$$

It is still left to show that the right-hand side of (60) reduces to the right-hand side of (23). To simplify the notations, we will denote $T=\left(M_{n}^{\prime}\right)^{-1}$. Since $\left(S^{*} M_{n}^{\prime}\right)^{\dagger}=P_{\mathcal{N} \perp\left(S^{*} M_{n}^{\prime}\right)}\left(S^{*} M_{n}^{\prime}\right)^{\dagger}$ and $P_{\mathcal{N} \perp}\left(S^{*} M_{n}^{\prime}\right)=P_{\left(T\left(\mathcal{S}^{\perp}\right)\right)^{\perp}}$ we rewrite $(60)$ as

$$
\mathbf{y}_{n+1}=\left(I-P_{T\left(\mathcal{S}^{\perp}\right)} P_{\mathcal{A}}\right)^{-1} P_{\left(T\left(\mathcal{S}^{\perp}\right)\right)^{\perp}}\left(S^{*} M_{n}^{\prime}\right)^{\dagger} r_{n} .
$$

Lemma 5: The transformation $F=I-P_{T\left(\mathcal{S}^{\perp}\right)} P_{\mathcal{A}}$ satisfies $F^{-1} P_{\left(T\left(\mathcal{S}^{\perp}\right)\right)^{\perp}}=P_{\mathcal{A}, T\left(\mathcal{S}^{\perp}\right)}$.

Proof: It is simple to see that for any $\mathbf{x} \in \mathcal{A}, F \mathbf{x}=$

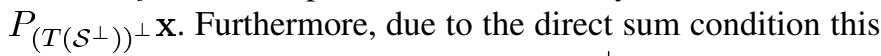
mapping is onto, that is $F(\mathcal{A})=\left(T\left(\mathcal{S}^{\perp}\right)\right)^{\perp}$. Also note that we can replace $P_{\left(T\left(\mathcal{S}^{\perp}\right)\right)^{\perp}}=P_{\left(T\left(\mathcal{S}^{\perp}\right)\right)^{\perp}, T\left(\mathcal{S}^{\perp}\right)}=P_{F(\mathcal{A}), T\left(\mathcal{S}^{\perp}\right)}$. Therefore

$$
\begin{aligned}
F^{-1} P_{\left(T\left(\mathcal{S}^{\perp}\right)\right)^{\perp}} & =F^{-1} P_{F \mathcal{A}, T\left(\mathcal{S}^{\perp}\right)} \\
& =F^{-1} F A\left(S^{*} T^{-1} F A\right)^{\dagger} S^{*} T^{-1} \\
& =A\left(S^{*} T^{-1} P_{\left.\left(T\left(\mathcal{S}^{\perp}\right)\right)^{\perp} A\right)^{\dagger} S^{*} T^{-1}}\right. \\
& =A\left(S^{*} T^{-1} A\right)^{\dagger} S^{*} T^{-1}=P_{\mathcal{A}, T\left(\mathcal{S}^{\perp}\right)}
\end{aligned}
$$

where we used $\mathcal{N}^{\perp}\left(S^{*} T^{-1}\right)=\left(T\left(\mathcal{S}^{\perp}\right)\right)^{\perp}$ in the transition to the last line.

Combining Lemma 5 with (61) and (27) yields that the POCS iterations reduce to

$\mathbf{y}_{n+1}=P_{\mathcal{A}, \mathcal{N}\left(S^{*} M_{n}^{\prime}\right)}\left(S^{*} M_{n}^{\prime}\right)^{\dagger}\left(S^{*} M_{n}^{\prime} \mathbf{y}_{n}+c-S^{*} M\left(\mathbf{y}_{n}\right)\right)$.
Now

$$
\begin{aligned}
& P_{\mathcal{A}, \mathcal{N}\left(S^{*} M_{n}^{\prime}\right)}\left(S^{*} M_{n}^{\prime}\right)^{\dagger} S^{*} \\
& \quad=A\left(S^{*} M_{n}^{\prime} A\right)^{\dagger} S^{*} M_{n}^{\prime}\left(S^{*} M_{n}^{\prime}\right)^{\dagger} S^{*} \\
& \quad=A\left(S^{*} M_{n}^{\prime} A\right)^{\dagger} P_{\mathcal{R}\left(S^{*} M_{n}^{\prime}\right)} S^{*} \\
& \quad=A\left(S^{*} M_{n}^{\prime} A\right)^{\dagger} S^{*}=\left(M_{n}^{\prime}\right)^{-1} P_{M_{n}^{\prime}(\mathcal{A}), \mathcal{S}^{\perp}}
\end{aligned}
$$

where we used $\mathcal{R}\left(S^{*} M_{n}^{\prime}\right)=\mathcal{R}\left(S^{*}\right)$ for bijective $M_{n}^{\prime}$ in the transition to the last line. Thus, we can rewrite (62) as

$$
\begin{aligned}
\mathbf{y}_{n+1} & =\left(M_{n}^{\prime}\right)^{-1} P_{M_{n}^{\prime}(\mathcal{A}), \mathcal{S}^{\perp}}\left(M_{n}^{\prime} \mathbf{y}_{n}+\mathbf{x}_{s}-M\left(\mathbf{y}_{n}\right)\right) \\
& =\mathbf{y}_{n}-\left(M_{n}^{\prime}\right)^{-1} P_{M_{n}^{\prime}(\mathcal{A}), \mathcal{S}^{\perp}}\left(M\left(\mathbf{y}_{n}\right)-\mathbf{x}_{s}\right)
\end{aligned}
$$

where we used $\mathbf{y}_{n} \in \mathcal{A}$ in the last equality.

\section{APPENDIX III \\ PROOF OF THEOREM 5}

Our proof will follow the geometric insight described by Fig. 5. For that matter we first define

$$
\theta_{1}=\arccos (\cos (\mathcal{A}, \mathcal{S})), \quad \theta_{2}=\arccos \left(\cos \left(\mathcal{A}, M_{n}^{\prime}(\mathcal{A})\right)\right)
$$

to be the maximal angles between the spaces, restricted to the interval $[0, \pi / 2]$. We now show that $\cos \left(M_{n}^{\prime}(\mathcal{A}), \mathcal{S}\right)$ and $\cos \left(\mathcal{S}, M_{n}^{\prime}(\mathcal{A})\right)$ are both lower bounded by $\cos \left(\theta_{1}+\theta_{2}\right)$. Then, we will derive a condition on the nonlinear distortion, assuring that $\cos \left(\theta_{1}+\theta_{2}\right)>0$.

Since, $\left\|P_{\mathcal{S}} \tilde{\mathbf{a}}\right\|_{L_{2}}=\left\|P_{\mathcal{S}} P_{\mathcal{A}} \tilde{\mathbf{a}}+P_{\mathcal{S}} P_{\mathcal{A}^{\perp}} \tilde{\mathbf{a}}\right\|_{L_{2}}$ we can lower bound

$$
\begin{aligned}
\left\|P_{\mathcal{S}} \tilde{\mathbf{a}}\right\|_{L_{2}} & \geq\left\|P_{\mathcal{S}} P_{\mathcal{A}} \tilde{\mathbf{a}}\right\|_{L_{2}}-\left\|P_{\mathcal{S}} P_{\mathcal{A}^{\perp}} \tilde{\mathbf{a}}\right\|_{L_{2}} \\
& \geq \cos (\mathcal{A}, \mathcal{S})\left\|P_{\mathcal{A}} \tilde{\mathbf{a}}\right\|_{L_{2}}-\sin \left(\mathcal{A}^{\perp}, \mathcal{S}^{\perp}\right)\left\|P_{\mathcal{A}^{\perp}} \tilde{\mathbf{a}}\right\|_{L_{2}} .
\end{aligned}
$$

We then have

$$
\begin{aligned}
& \cos \left(M_{n}^{\prime}(\mathcal{A}), \mathcal{S}\right) \\
& =\inf _{\tilde{\mathbf{a}} \in M_{n}^{\prime}(\mathcal{A}),\|\tilde{\mathbf{a}}\|_{L_{2}}=1}\left\|P_{\mathcal{S}} \tilde{\mathbf{a}}\right\|_{L_{2}} \\
& \geq \cos (\mathcal{A}, \mathcal{S}) \inf _{\tilde{\mathbf{a}} \in M_{n}^{\prime}(\mathcal{A}),\|\tilde{\mathbf{a}}\|_{L_{2}}=1}\left\|P_{\mathcal{A}} \tilde{\mathbf{a}}\right\|_{L_{2}} \\
& \quad-\sin \left(\mathcal{A}^{\perp}, \mathcal{S}^{\perp}\right) \sup _{\tilde{\mathbf{a}} \in M_{n}^{\prime}(\mathcal{A}),\|\tilde{\mathbf{a}}\|_{L_{2}}=1}\left\|P_{\mathcal{A}}{ }^{\perp} \tilde{\mathbf{a}}\right\|_{L_{2}} \\
& \quad \\
& \quad \cos (\mathcal{A}, \mathcal{S}) \cos \left(M_{n}^{\prime}(\mathcal{A}), \mathcal{A}\right)-\sin (\mathcal{S}, \mathcal{A}) \sin \left(M_{n}^{\prime}(\mathcal{A}), \mathcal{A}\right)
\end{aligned}
$$

where we used $\sin \left(\mathcal{A}^{\perp}, \mathcal{S}^{\perp}\right)=\sin (\mathcal{S}, \mathcal{A})$ in the last equality. The condition $\mathcal{A} \oplus \mathcal{S}^{\perp}=L_{2}$ also implies [16] $\sin (\mathcal{S}, \mathcal{A})=\sin (\mathcal{A}, \mathcal{S})$. Similarly, $M_{n}^{\prime}(\mathcal{A}) \oplus \mathcal{A}^{\perp}=L_{2}$ implies $\sin \left(M_{n}^{\prime}(\mathcal{A}), \mathcal{A}\right)=\sin \left(\mathcal{A}, M_{n}^{\prime}(\mathcal{A})\right)$ and $\cos \left(M_{n}^{\prime}(\mathcal{A}), \mathcal{A}\right)=$ $\cos \left(\mathcal{A}, M_{n}^{\prime}(\mathcal{A})\right)$. Thus, we end up with

$\cos \left(M_{n}^{\prime}(\mathcal{A}), \mathcal{S}\right) \geq \cos (\mathcal{A}, \mathcal{S}) \cos \left(\mathcal{A}, M_{n}^{\prime}(\mathcal{A})\right)$

$$
-\sin (\mathcal{A}, \mathcal{S}) \sin \left(\mathcal{A}, M_{n}^{\prime}(\mathcal{A})\right) \text {. }
$$


Interpreting (65) in terms of the angles (63) we can rewrite (65) as

$$
\cos \left(M_{n}^{\prime}(\mathcal{A}), \mathcal{S}\right) \geq \cos \left(\theta_{1}+\theta_{2}\right)
$$

Thus, as long as $\cos \left(\theta_{1}+\theta_{2}\right)>0$ i.e., $\theta_{1}+\theta_{2}<\pi / 2$ we also have $\cos \left(M_{n}^{\prime}(\mathcal{A}), \mathcal{S}\right)>0$

In a similar manner we can show that

$$
\begin{aligned}
\cos \left(\mathcal{S}, M_{n}^{\prime}(\mathcal{A})\right) \geq & \cos (\mathcal{A}, \mathcal{S}) \cos \left(M_{n}^{\prime}(\mathcal{A}), \mathcal{A}\right) \\
& -\sin \left(\mathcal{A}^{\perp}, \mathcal{S}^{\perp}\right) \sin \left(M_{n}^{\prime}(\mathcal{A}), \mathcal{A}\right) \\
= & \cos (\mathcal{A}, \mathcal{S}) \cos \left(\mathcal{A}, M_{n}^{\prime}(\mathcal{A})\right) \\
& -\sin (\mathcal{A}, \mathcal{S}) \sin \left(\mathcal{A}, M_{n}^{\prime}(\mathcal{A})\right)
\end{aligned}
$$

where we used $\mathcal{A} \oplus \mathcal{S}^{\perp}=L_{2}, M_{n}^{\prime}(\mathcal{A}) \oplus \mathcal{A}^{\perp}=L_{2}$ in the last equality. Since the lower bounds in (65) and (67) are the same, $\cos \left(\theta_{1}+\theta_{2}\right)>0$ implies $M_{n}^{\prime}(\mathcal{A}) \oplus \mathcal{S}^{\perp}=L_{2}$, as required.

Finally, we interpret the condition $\cos \left(\theta_{1}+\theta_{2}\right)>0$ in terms of amplitude bounds for $\mathbf{m}_{n}^{\prime}(t)$. Starting from the requirement

$$
\cos (\mathcal{A}, \mathcal{S}) \cos \left(\mathcal{A}, M_{n}^{\prime}(\mathcal{A})\right)-\sin (\mathcal{A}, \mathcal{S}) \sin \left(\mathcal{A}, M_{n}^{\prime}(\mathcal{A})\right)>0
$$

it is a matter of straightforward calculations to show that (68) is equivalent to $\cos \left(\mathcal{A}, M_{n}^{\prime}(\mathcal{A})\right)>\sin (\mathcal{A}, \mathcal{S})$. Using the lower bound in (40), it is thus sufficient to show that

$$
\frac{1-\left\|1-\mathbf{m}_{n}^{\prime}\right\|_{L_{\infty}}}{1+\left\|1-\mathbf{m}_{n}^{\prime}\right\|_{L_{\infty}}}>\sin (\mathcal{A}, \mathcal{S})
$$

which is equivalent to (42).

\section{APPENDIX IV \\ PROOF OF GRADIENT LIPSCHITZ CONTINUITY}

Using $\nabla f(a)=\left(S^{*} M_{n}^{\prime} A\right)^{*} e(a)$ and denoting $Q_{n}=$ $S^{*} M_{n}^{\prime} A, e_{n}=e\left(a_{n}\right)$,

$$
\begin{aligned}
& \| \nabla \\
& \left.\quad=\| a_{m}\right)-\nabla f\left(a_{n}\right) \|_{l_{2}} \\
& \quad=\frac{1}{2}\left\|\left(Q_{m}^{*}-Q_{n}^{*}\right)\left(e_{m}+e_{n}\right)+\left(Q_{m}^{*}+Q_{n}^{*}\right)\left(e_{m}-e_{n}\right)\right\|_{l_{2}} \\
& \quad \leq \frac{1}{2}\left\|Q_{m}-Q_{n}\right\|\left\|e_{m}+e_{n}\right\|_{l_{2}}+\frac{1}{2}\left\|Q_{m}+Q_{n}\right\|\left\|e_{m}-e_{n}\right\|_{l_{2}} .
\end{aligned}
$$

Letting $U_{M^{\prime}}$ to be the Lipschitz bound of $\mathbf{m}^{\prime}$ we have

$$
\begin{aligned}
& \left\|M_{m}^{\prime}-M_{n}^{\prime}\right\| \\
& \quad=\sup _{\|\mathbf{f}\|_{L_{2}}=1}\left\|\left(M_{m}^{\prime}-M_{n}^{\prime}\right) \mathbf{f}\right\|_{L_{2}} \\
& =\sup _{\|\mathbf{f}\|_{L_{2}}=1}\left(\int\left|\left(\mathbf{m}_{m}^{\prime}(t)-\mathbf{m}_{n}^{\prime}(t)\right) \mathbf{f}(t)\right|^{2} d t\right)^{1 / 2} \\
& \leq \sup _{\|\mathbf{f}\|_{L_{2}}=1}\left(\int U_{M^{\prime}}^{2}\left|\left(\mathbf{y}_{m}(t)-\mathbf{y}_{n}(t)\right) \mathbf{f}(t)\right|^{2} d t\right)^{1 / 2} \\
& =U_{M^{\prime}}\left\|\mathbf{y}_{m}-\mathbf{y}_{n}\right\|_{L_{2}} .
\end{aligned}
$$

Hence, by (2) and (5)

$$
\begin{aligned}
\left\|Q_{m}-Q_{n}\right\| & =\left\|S^{*}\left(M_{m}^{\prime}-M_{n}^{\prime}\right) A\right\| \\
& \leq U_{S} U_{M^{\prime}}\left\|\mathbf{y}_{m}-\mathbf{y}_{n}\right\|_{L_{2}} U_{A} .
\end{aligned}
$$

Utilizing (72) and $\left\|Q_{m}+Q_{n}\right\| \leq\left\|Q_{m}\right\|+\left\|Q_{n}\right\| \leq 2 U_{S} U_{g} U_{A}$ we can further upper bound (70) by

$$
\begin{aligned}
\| \nabla & f\left(a_{m}\right)-\nabla f\left(a_{n}\right) \|_{l_{2}} \\
\leq & \frac{1}{2} U_{S} U_{M^{\prime}}\left\|\mathbf{y}_{m}-\mathbf{y}_{n}\right\|_{L_{2}} U_{A}\left\|e_{m}+e_{n}\right\|_{l_{2}} \\
& +U_{S} U_{g} U_{A}\left\|e_{m}-e_{n}\right\|_{l_{2}} .
\end{aligned}
$$

Since the direction $p_{n}$ is gradient related, and due to the choice of the step size, the sequence $\left\{\left\|e_{n}\right\|_{l_{2}}\right\}$ is non ascending. Thus, we can upper bound $\left\|e_{m}+e_{n}\right\|_{l_{2}} \leq 2\left\|e_{0}\right\|_{l_{2}}<\infty$. The latter also equals $2\|c\|_{l_{2}}$, when we assume $\mathbf{m}(0)=0$.

Finally, using (46) and a derivation similar to (3) to show that $M$ is Lipschitz continuous with upper bound $U_{g}$, we obtain $\left\|e_{m}-e_{n}\right\|_{l_{2}} \leq U_{S} U_{g} U_{A}\left\|a_{m}-a_{n}\right\|_{l_{2}}$. In total, this leads to Lipschitz continuity of $\nabla f$ as desired:

$$
\begin{aligned}
& \left\|\nabla f\left(a_{m}\right)-\nabla f\left(a_{n}\right)\right\|_{l_{2}} \\
& \quad \leq U_{S} U_{M^{\prime}} U_{A}^{2}\left\|a_{m}-a_{n}\right\|_{l_{2}}\|c\|_{l_{2}}+U_{S}^{2} U_{g}^{2} U_{A}^{2}\left\|a_{m}-a_{n}\right\|_{l_{2}} \\
& \quad=U_{S} U_{A}^{2}\left(U_{M^{\prime}}\|c\|_{l_{2}}+U_{S} U_{g}^{2}\right)\left\|a_{m}-a_{n}\right\|_{l_{2}} .
\end{aligned}
$$

\section{REFERENCES}

[1] S. Kawai, M. Morimoto, N. Mutoh, and N. Teranishi, "Photo response analysis in CCD image sensors with a VOD structure," IEEE Trans. Electron Devices, vol. 42, no. 4, pp. 652-655, 1995.

[2] G. C. Holst, CCD Arrays Cameras and Displays. Bellingham, WA: SPIE Optical Engineering Press, 1996.

[3] F. Pejović and D. Maksimović, "A new algorithm for simulation of power electronic systems using piecewise-linear device models," IEEE Trans. Power Electron., vol. 10, no. 3, pp. 340-348, 1995.

[4] H. Farid, "Blind inverse gamma correction," IEEE Trans. Image Process., vol. 10, pp. 1428-1433, Oct. 2001.

[5] K. Shafique and M. Shah, "Estimation of radiometric response functions of a color camera from differently illuminated images," in Proc. Int. Conf. Image Processing (ICIP), 2004, pp. 2339-2342.

[6] K. Kose, K. Endoh, and T. Inouye, "Nonlinear amplitude compression in magnetic resonance imaging: Quantization noise reduction and data memory saving," IEEE Aerosp. Electron. Syst. Mag., vol. 5, no. 6, pp. 27-30, Jun. 1990.

[7] C. H. Tseng, "Bandpass sampling criteria for nonlinear systems," IEEE Trans. Signal Process., vol. 50, no. 3, pp. 568-577, Mar. 2002.

[8] Y. M. Zhu, "Generalized sampling theorem," IEEE Trans. Circuits Syst. II, vol. 39, no. 8, pp. 587-588, Aug. 1992.

[9] M. Unser and A. Aldroubi, "A general sampling theory for nonideal acquisition devices," IEEE Trans. Signal Process., vol. 42, no. 11, pp. 2915-2925, Nov. 1994.

[10] Y. C. Eldar, "Sampling and reconstruction in arbitrary spaces and oblique dual frame vectors," J. Fourier Anal. Appl., vol. 1, no. 9, pp. 77-96, Jan. 2003.

[11] M. Unser and J. Zerubia, "Generalized sampling: Stability and performance analysis," IEEE Trans. Signal Process., vol. 45, no. 12, pp. 2941-2950, Dec. 1997.

[12] Y. C. Eldar and T. G. Dvorkind, "A minimum squared-error framework for generalized sampling," IEEE Trans. Signal Process., vol. 54, no. 6, pp. 2155-2167, Jun. 2006.

[13] Y. C. Eldar, "Sampling without input constraints: Consistent reconstruction in arbitrary spaces," in Sampling, Wavelets and Tomography, A. I. Zayed and J. J. Benedetto, Eds. Boston, MA: Birkhäuser, 2004, pp. 33-60. 
[14] Y. C. Eldar and T. Werther, "General framework for consistent sampling in Hilbert spaces," Int. J. Wavelets, Multires., Inf. Process., vol. 3, no. 3, pp. 347-359, Sep. 2005.

[15] R. T. Behrens and L. L. Scharf, "Signal processing applications of oblique projection operators," IEEE Trans. Signal Process., vol. 42, no. 6, pp. 1413-1424, Jun. 1994.

[16] W. S. Tang, "Oblique projections, biorthogonal Riesz bases and multiwavelets in Hilbert space," Proc. Amer. Math. Soc., vol. 128, no. 2, pp. 463-473, 2000.

[17] H. H. Bauschke, J. M. Borwein, and A. S. Lewis, "The method of cyclic projections for closed convex sets in Hilbert space," Contemp. Math., vol. 204, pp. 1-38, 1997.

[18] J. Nocedal and S. J. Wright, Numerical Optimization. New York: Springer, 1999.

[19] D. P. Bertsekas, Nonlinear Programming, Second ed. Belmont, MA: Athena Scientific, 1999.

[20] O. Christensen, H. O. Kim, R. Y. Kim, and J. K. Lim, "Perturbation of frame sequences in shift-invariant spaces," The J. Geometric Anal., vol. 15, no. 2, 2005.

[21] F. J. Doyle, III, R. K. Pearson, and B. A. Ogunnaike, Identification and Control Using Volterra Models. Berlin, Germany: Springer, 2002.

[22] A. Ben-Israel and T. N. E. Greville, Generalized Inverses: Theory and Applications. New York: Wiley, 1974.

[23] O. Christensen, An Introduction to Frames and Riesz Bases. Boston, MA: Birkhäuser, 2003.

[24] M. S. Berger, Nonlinearity and Functional Analysis. New York: Academic, 1977.

[25] O. Christensen and Y. C. Eldar, "Oblique dual frames and shift-invariant spaces," Appl. Comput. Harmon. Anal., vol. 17, no. 1, pp. 48-68, 2004.

[26] P. P. Vaidyanathan, "Generalizations of the sampling theorem: Seven decades after Nyquist," IEEE Trans. Circuit Syst. I, vol. 48, no. 9, pp. 1094-1109, Sep. 2001.

[27] A. Levi and H. Stark, "Signal restoration from phase by projections onto convex sets," in Proc. IEEE. Int. Conf. Acoustics, Speech, Signal Processing (ICASSP), Boston, 1983, vol. 8, pp. 1458-1460.

[28] E. Yudilevich, A. Levi, G. J. Habetler, and H. Stark, "Restoration of signals from their signed Fourier-transform magnitude by the method of generalized projections," J. Opt. Soc. Amer. A, vol. 4, no. 1, pp. 236-246, Jan. 1987.

[29] O. Christensen, "Operators with closed range, pseudo-inverses, and perturbation of frames for a subspace," Canad. Math. Bull., vol. 42, no. 1, pp. 37-45, 1999.

[30] G. R. Walsh, Methods of Optimization. New York: Wiley, 1975.

[31] N. Aronszajn, "Theory of reproducing kernels," Trans. Amer. Math. Soc., vol. 68, no. 3, pp. 337-404, May 1950.

[32] T. Ando, "Reproducing kernel spaces and quadratic inequalities," Hokkaido Univ., Research Inst. of Applied Electricity, Sapporo, Japan, 1987, Lecture Notes.

[33] M. Unser, "Sampling—50 years after Shannon," IEEE Proc., vol. 88, pp. 569-587, Apr. 2000.

[34] A. Zeitouny, Z. Tamir, A. Feldster, and M. Horowitz, "Optical sampling of narrowband microwave signals using pulses generated by electroabsorption modulators," Elsevier Opt. Commun., no. 256, pp. 248-255, 2005.

[35] A. Yariv, Quantum Electronics. New York: Wiley, 1988.

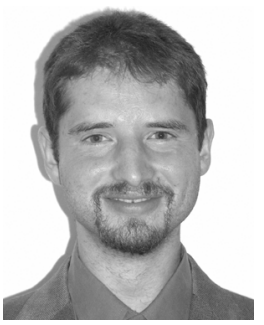

Tsvi G. Dvorkind received the B.Sc. degree (summa cum laude) in computer engineering in 2000 , the M.Sc. degree (summa cum laude) in electrical engineering in 2003, and the Ph.D. degree in electrical engineering in 2007, all from the Technion-Israel Institute of Technology, Haifa, Israel.

From 1998 to 2000 he worked at the Electro-Optics Research \& Development Company at the Technion, and during 2000-2001 at the Jigami Corporation. He is now with the Rafael Company, Haifa, Israel. His research interests include speech enhancement and acoustical localization, general parameter estimation problems, and sampling theory.

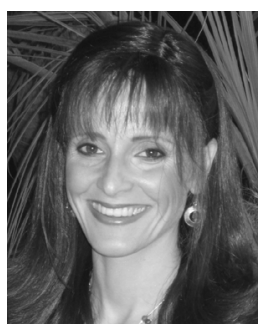

Yonina C. Eldar (S'98-M'02-SM'07) received the B.Sc. degree in physics and the B.Sc. degree in electrical engineering, both from Tel-Aviv University (TAU), Tel-Aviv, Israel, in 1995 and 1996, respectively, and the Ph.D. degree in electrical engineering and computer science from the Massachusetts Institute of Technology (MIT), Cambridge, in 2001.

From January 2002 to July 2002, she was a Postdoctoral Fellow at the Digital Signal Processing Group at MIT. She is currently an Associate Professor in the Department of Electrical Engineering at the Technion-Israel Institute of Technology, Haifa, Israel. She is also a Research Affiliate with the Research Laboratory of Electronics at MIT. Her research interests are in the general areas of signal processing, statistical signal processing, and computational biology.

Dr. Eldar was in the program for outstanding students at TAU from 1992 to 1996. In 1998, she held the Rosenblith Fellowship for study in electrical engineering at MIT, and in 2000, she held an IBM Research Fellowship. From 2002 to 2005, she was a Horev Fellow of the Leaders in Science and Technology program at the Technion and an Alon Fellow. In 2004, she was awarded the Wolf Foundation Krill Prize for Excellence in Scientific Research, in 2005 the Andre and Bella Meyer Lectureship, in 2007 the Henry Taub Prize for Excellence in Research, and in 2008 the Hershel Rich Innovation Award, the Award for Women with Distinguished Contributions, and the Muriel \& David Jacknow Award for Excellence in Teaching. She is a member of the IEEE Signal Processing Theory and Methods Technical Committee, an Associate Editor for the IEEE TRANSACTIONS ON Signal PROCESSING, the EURASIP Journal of Signal Processing, and the SIAM Journal on Matrix Analysis and Applications, and on the Editorial Board of Foundations and Trends in Signal Processing.

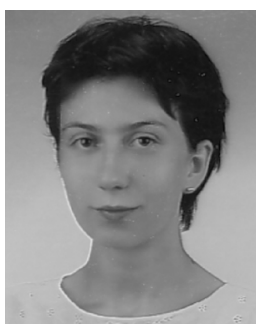

Ewa Matusiak received the Master's degree in mathematics and electrical engineering from Oklahoma University, Norman, in 2003 and the Ph.D. degree in mathematics from the University of Vienna in 2007.

Since then, she has been a Postdoctoral Researcher at the Faculty of Mathematics, University of Vienna. Her interests lie in Gabor analysis, especially on finite Abelian groups, uncertainty principle, and wireless communication. 